\title{
RELATIVE VIRTUAL LOCALIZATION AND VANISHING OF TAUTOLOGICAL CLASSES ON MODULI SPACES OF CURVES
}

\author{
TOM GRABER AND RAVI VAKIL
}

\begin{abstract}
We prove a localization formula for the moduli space of stable relative maps. As an application, we prove that all codimension $i$ tautological classes on the moduli space of stable pointed curves vanish away from strata corresponding to curves with at least $i-$ $g+1$ genus 0 components. As consequences, we prove and generalize various conjectures and theorems about various moduli spaces of curves (due to Diaz, Faber, Getzler, Ionel, Looijenga, Pandharipande, and others). This theorem appears to be the geometric content behind these results; the rest is straightforward graph combinatorics. The theorem also suggests the importance of the stratification of the moduli space by number of rational components.
\end{abstract}

\section{CONTENTS}

1. Introduction 1

2. Stable relative maps 3

3. Relative virtual localization 10

4. Vanishing of tautological classes on moduli spaces of curves and stratification by number of rati

5. Applications of Theorem $\star$

References

\section{INTRODUCTION}

"Relative virtual localization" (Theorem 3.6) is a localization formula for the moduli space of stable relative maps, using the formalism of [GrPa1]. It is straightforward to postulate the form of such a formula. Proving it requires three key ingredients.

(A) The moduli space is shown to admit a $\mathbb{C}^{*}$-equivariant locally closed immersion into a smooth Deligne-Mumford stack.

(B) The natural virtual fundamental class on the $\mathbb{C}^{*}$-fixed locus is shown to arise from the $\mathbb{C}^{*}$-fixed part of the obstruction theory of the moduli space.

(C) The virtual normal bundle to the fixed locus is determined.

Date: December 20, 2004.

The first author is partially supported by NSF Grant DMS-0301179 and an Alfred P. Sloan Research Fellowship. The second author is partially supported by NSF Grant DMS-0238532, an AMS Centennial Fellowship, and an Alfred P. Sloan Research Fellowship.

2000 Mathematics Subject Classification: Primary 14H10, 14D22, Secondary 14C15, 14F43. 
The technical hypothesis (A) is presumably unnecessary, but the reader is warned that it has as yet not been excised from the proof of virtual localization [GrPa1]. In verifying it, we exhibit the moduli space as a global quotient (Sect. 2.6), which may be independently useful.

We use J. Li's algebro-geometric definition of this moduli space, and his description of its obstruction theory [Li1, [Li2]. We note the earlier definitions of relative stable maps in the differentiable category due to A-M. Li and Y. Ruan, and Ionel and Parker, [LR, IP1. [IP2], as well as Gathmann's work in the algebraic category in genus 0 [Ga1]. We give two proofs of relative virtual localization, which we hope will give the reader insight into the technicalities of the moduli space of relative stable maps and its properties.

The tautological ring of the moduli space of stable $n$-pointed curves genus $g$ curves $\overline{\mathcal{M}}_{g, n}$ (and other partial compactifications of the moduli space of curves $\mathcal{M}_{g, n}$ ) is a subring of the Chow ring (with rational coefficients), containing (informally) "all of the classes naturally arising in geometry". As an application of relative virtual localization, we prove the following result, announced in [Va3].

1.1. Theorem $\star$. - Any tautological class of codimension $i$ on $\overline{\mathcal{M}}_{g, n}$ vanishes on the open set consisting of strata satisfying

$$
\text { \# genus } 0 \text { components }<i-g+1 \text {. }
$$

(We recall the definition of tautological classes in Section 4) Equivalently, any tautological class of codimension $i$ is the pushforward of a class supported on the locus where the number of genus 0 components is at least $i-g+1$. The corresponding result is not true for Chow classes in general; $(g, n, i)=(1,11,11)$ provides a counterexample (see [GrVa2, Rem. 1.1]).

We emphasize that the proof of Theorem $\star$ is quite short and naive. (This portion of the paper may be read independently of the others, assuming only the form of relative virtual localization.) We define Hurwitz classes in the Chow group of the moduli space of curves as the pushforwards of the (virtual) class of maps to $\mathbb{P}^{1}$ with some branch points fixed. There are a very small number of tools in the Gromov-Witten theorist's toolkit, and we employ two of them. By deforming the target $\mathbb{P}^{1}$, we show that Hurwitz classes lie in a deep stratum where the curve has many genus 0 components. Using (relative virtual) localization, we express tautological classes as linear combinations of Hurwitz classes, using the "polynomiality trick" of [GrVa2].

In Section [5 we give numerous consequences of Theorem $\star$, proving many "vanishing" conjectures and theorems on moduli spaces of curves (due to Diaz, Faber, Getzler, Ionel, Looijenga, Pandharipande, and others). This section may also be read independently of the others. Theorem $\star$ implies these various results via straightforward graph combinatorics, and in most cases extends them. The morals of Theorem $\star$ seem to be: $i$ ) this result is the fundamental geometry behind these results, and $i i)$ the strange stratification of the moduli space of curves by number of rational components may be potentially important. In particular, Theorem $\star$ raises some natural questions. For example, Looijenga 
asks if this " $\geq k$-rational component locus" has the pseudo-convexity property of being the union of $k+g-1$ affine open sets. This would imply the Diaz-type results of Section 5.9 for example. See also [HaLo, Prob. 6.5] for topological and cohomological vanishing consequences.

We expect relative virtual localization to have many applications; some of these consequences have already appeared. For example, as described in [GrVa1, Sect. 5], it gives an immediate proof of the "ELSV formula" [ELSV1, ELSV2, GrVa1]. A. Gathmann has computed the genus 1 Gromov-Witten invariants of the quintic threefold using relative virtual localization [Ga2]. Further applications are in [FbPa2] (including different proofs of some of the results of Section [5), and [LLZ1, LLZ2]. Relative virtual localization also plays an essential role in Okounkov and Pandharipande's proof of the Virasoro constraints for target curve [OPa].

Throughout this paper we work over $\mathbb{C}$, or any other algebraically closed field of characteristic 0 . Our methods are purely algebraic.

1.2. Acknowledgments. We are grateful to J. Li, R. Pandharipande, E.-N. Ionel, A. Gathmann, and Y. Ruan for useful conversations, and to C. Faber for inspiration. Most of this work was carried out when the authors were at Harvard University and M.I.T. respectively, and it is a pleasure to acknowledge these institutions here.

\section{STABLE RELATIVE MAPS}

In order to make this paper as self-contained as possible, we review relevant background on the moduli space of stable relative maps. We give J. Li's algebro-geometric definition of stable relative maps and their moduli stack. (We again emphasize the prior work of Li-Ruan and Ionel-Parker in the differentiable category.) We then define stable relative maps to a non-rigid target (implicit in [Li1] and the earlier work in the differentiable category). We describe the moduli space of targets $\mathcal{T}$ and $\mathcal{T}_{\sim}$. We then show that the moduli space of stable relative maps satisfies the technical hypothesis (A) of virtual localization, by exhibiting it as a certain global quotient. Finally, we give a description of the perfect obstruction theory of the moduli space in terms of a relative obstruction theory over the moduli stack of source and target, which is a useful description for applications, including the proof of Theorem $\star$ in Section 4 ,

2.1. Notation. We first set notation for the next two sections. Suppose that $X$ is a smooth complex projective variety, and $D$ is a smooth divisor. Let $Y$ be the $\mathbb{P}^{1}$-bundle over $D$ corresponding to $N_{D / X} \oplus \mathcal{O}_{D}: Y=\mathbb{P}_{D}\left(N_{D / X} \oplus \mathcal{O}_{D}\right)$. The bundle $Y$ has two natural disjoint sections, one with normal bundle $N_{D / X}^{\vee}$ and the other with normal bundle $N_{D / X}$; call these the zero section and the infinity section of $Y$ respectively. For any non-negative integer $l$, define $Y_{l}$ by gluing together $l$ copies of $Y$, where the infinity section of the $i^{\text {th }}$ component is glued to the zero section of the $(i+1)^{\text {st }}(1 \leq i<l)$. Denote the zero section of the $i^{\text {th }}$ component by $D_{i-1}$, and the infinity section by $D_{i}$, so Sing $Y_{l}=\cup_{i=1}^{l-1} D_{i}$. We will also denote $D_{l}$ by $D_{\infty}$. Define $X_{l}$ by gluing $X$ along $D$ to $Y_{l}$ along $D_{0}$. (Thus for 
example Sing $X_{l}=\cup_{i=0}^{l-1} D_{i}$, and $X_{0}=X$.) Let Aut $_{D} Y_{l} \cong\left(\mathbb{C}^{*}\right)^{l}$ be the obvious group of automorphisms of $Y_{l}$ preserving $D_{0}, D_{\infty}$, and the morphism to $D$ and let Aut $_{D} X_{l}$ be the group of automorphisms of $X_{l}$ preserving $X$ (and $D$ ) and with restriction to $Y_{l}$ contained in $\operatorname{Aut}_{D} Y_{l}$ (so Aut $\left.X_{l} \equiv \operatorname{Aut}_{D} Y_{l} \cong\left(\mathbb{C}^{*}\right)^{l}\right)$. Note that if $D$ is disconnected, these conventions differ from the most natural interpretation of the notation which would be strictly larger groups. This will be irrelevant for the applications in the sequel, but important in general.

2.2. The stack of stable relative maps. We recall the definition of stable relative maps to $X$ relative to a divisor $D$. If $\Gamma$ denotes the data of

A1. arithmetic genus $g$ of the source curve,

A2. element $\beta$ of $H_{2}(X)$,

A3. number $n$ of marked points mapped to $D$, and corresponding partition $\alpha$ of $\beta \cdot D$ into $n$ parts, $\alpha_{1}, \ldots, \alpha_{n}$, and

A4. number $m$ of other marked points,

we denote the moduli stack by $\overline{\mathcal{M}}_{\Gamma}(X, D)$.

We make two conventions about stable relative maps to $X$ relative to a divisor $D$ differing from Li's: the source curve need not be connected, and the points mapping to $D$ are labeled. These conventions are not mathematically important, but simplify the exposition. (Hence for us, moduli spaces of stable relative maps are not in general connected, and the arithmetic genus of the source curve may be negative.)

The $\mathbb{C}$-points of this stack correspond to morphisms $C \stackrel{f}{\rightarrow} X_{l} \rightarrow X$ where $C$ is a nodal curve of arithmetic genus $g$ equipped with a set of marked smooth points, $p_{1}, \ldots, p_{m}$, $q_{1}, \ldots, q_{n}$. The morphism $f$ has the property that $f^{*}\left(D_{\infty}\right)=\sum \alpha_{i} q_{i}$, and it satisfies the predeformability condition above the singular locus Sing $X_{l}=\cup_{i=0}^{l-1} D_{i}$ of $X_{l}$, meaning that the preimage of the singular locus is a union of nodes of $C$, and if $p$ is one such node, then the two branches of $C$ at $p$ map into different irreducible components of $X_{l}$, and their orders of contact with the divisor $D_{i}$ (in their respective components of $X_{l}$ ) are equal. We will refer to these nodes as the distinguished nodes of $C$. The morphism $f$ is also required to satisfy a stability condition that there are no infinitesimal automorphisms of the sequence of maps $\left(C, p_{1}, \ldots, p_{m}, q_{1}, \ldots, q_{n}\right) \rightarrow X_{l} \rightarrow X$, where the allowed automorphisms of the map from $X_{l}$ to $X$ are $\operatorname{Aut}_{D}\left(X_{l}\right)$.

2.3. The paper [Li1] defines a good notion of a family of such maps, i.e. a moduli functor or groupoid. A family of stable relative maps over a base scheme $S$ is a pair of morphisms of flat $S$-schemes $C \stackrel{f}{\rightarrow} \bar{X} \rightarrow X \times S$, where for each $\mathbb{C}$-point $s$ in $S$, the fiber $C_{s} \stackrel{f_{s}}{\rightarrow}$ $\bar{X}_{s} \rightarrow X$ gives a stable relative map. There is also the predeformability condition, that in a neighborhood of a node of $C_{s}$ mapping to a singularity of $\bar{X}_{s}$, we can choose étalelocal coordinates on $S, C$, and $\bar{X}$ with charts of the form $\operatorname{Spec} R$, Spec $R[u, v] / u v=b$ and Spec $R\left[x, y, z_{1}, \ldots, z_{k}\right] / x y=a$ respectively, with the map of the form $x \mapsto \alpha u^{m}, y \mapsto \beta v^{m}$ with $\alpha$ and $\beta$ units, and no restriction on the $z_{i}$. Given any family of stable morphisms to 
an allowed family of target schemes, the locus of maps satisfying this predeformability condition naturally forms a locally closed subscheme.

2.4. Stable relative maps to a non-rigid target. We make explicit the following variation on stable relative maps. Such maps appear in the boundary of the space of "usual" stable relative maps [Li1, Sect. 3.1]. (For this definition, $Y$ can be any $\mathbb{P}^{1}$-bundle with two disjoint sections over a smooth variety $D$, but we will be using this definition in the context described in Section 2.1])

We consider morphisms to $\left(Y_{l}, D_{0} \cup D_{\infty}\right)$, where two stable maps

$$
\left(C, p_{1}, \ldots, p_{m}, q_{1}, \ldots, q_{n}, r_{1}, \ldots, r_{n^{\prime}}\right) \longrightarrow\left(Y_{l}, D_{0}, D_{\infty}\right)
$$

$\left(r_{i} \rightarrow D_{0}, q_{i} \rightarrow D_{\infty}\right)$ and

$$
\left(C^{\prime}, p_{1}^{\prime}, \ldots, p_{m}^{\prime}, q_{1}^{\prime}, \ldots, q_{n}^{\prime}, r_{1}^{\prime}, \ldots, r_{n^{\prime}}^{\prime}\right) \longrightarrow\left(Y_{l}, D_{0}, D_{\infty}\right)
$$

are considered isomorphic if there is a commutative diagram

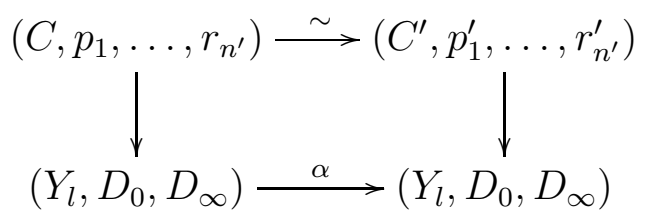

with $\alpha \in \operatorname{Aut}_{D} Y_{l}$. Predeformability is required above the singularities of $Y_{l}$. We will call these stable relative maps to a non-rigid target. The definition of a family of such maps is the obvious variation of that for stable relative maps described in the previous section (see also [Li1, Sect. 2.2]).

Such maps also form a Deligne-Mumford moduli stack. If $\Gamma$ denotes the data of

B1. arithmetic genus of the source curve,

B2. element $\beta$ of $H_{2}(Y)$,

B3. number of marked points mapped to $D_{0}$ (resp. $\left.D_{\infty}\right)$, and corresponding partition of $\beta \cdot D_{0}$ (resp. $\left.D_{\infty}\right)$, and

B4. number of other marked points,

we denote the corresponding moduli stack $\overline{\mathcal{M}}_{\Gamma}\left(Y, D_{0}, D_{\infty}\right)_{\sim}$. This stack may be constructed by a straightforward variation of the global quotient construction of Section 2.6

2.5. The moduli spaces of targets $\mathcal{T}$ and $\mathcal{T}_{\sim}$. Let $\mathcal{T}$ be the Artin stack parametrizing the possible targets of relative stable maps to $(X, D)$. (This is called the stack of expanded degenerations in [Li1, Sect. 1].) It has one $\mathbb{C}$-point for each nonnegative integer. $\mathcal{T}$ is isomorphic to the open substack of the Artin stack $\mathfrak{M}_{0,3}$ of prestable 3-pointed genus 0 curves consisting of curves where the only nodes separate point $\infty$ from points 0 and 1 (see Figure 1). On the level of $\mathbb{C}$-points, this open immersion corresponds to replacing $X_{l}$ with a copy of $\mathbb{P}^{1}$ (with 0 and 1 marked) attached at $\infty$ to the fiber of $Y_{l}$ over a fixed point of $D$, marking the point on $D_{\infty}$. The equality of these stacks can be seen either by describing this construction for families (using a blow-up construction rather than gluing), or by 


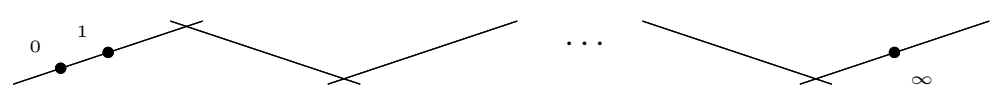

FIGURE 1. The open substack $\mathcal{T}$ of $\overline{\mathfrak{M}}_{0,3}$ : all nodes separate $\infty$ from 0 and 1

noting that the construction in [Li1] is independent of $(X, D)$. Note in particular that $\mathcal{T}$ is independent of $(X, D)$, but the universal family is not. We refer the reader to [Li1] for the construction of the universal family over $\mathcal{T}$.

The moduli space $\overline{\mathcal{M}}_{\Gamma}(X, D)$ is then simply the locally closed subset of the space of stable morphisms to the universal family over $\mathcal{T}$ where the predeformability and stability conditions are satisfied.

The analogous space for stable relative maps to non-rigid targets is the stack $\mathcal{T}_{\sim}$ which is the open substack of $\mathfrak{M}_{0,2}$ parametrizing 2-pointed curves where the only nodes separate 0 from $\infty$. The line bundle corresponding to the cotangent space at the point 0 will play an important role; we denote its first Chern class by $\psi$. (The notation $\psi_{0}$ is more usual, but we wish to avoid confusing this class with the $\psi$-classes coming from the marked points on the source of the relative stable map.) We denote the pullback of $\psi$ to $\overline{\mathcal{M}}_{\Gamma}\left(Y, D_{0}, D_{\infty}\right) \sim$ by $\psi$ as well. See [Ka] for a different definition of $\psi$, and a more detailed discussion.

2.6. Global equivariant embedding, and $\overline{\mathcal{M}}_{\Gamma}(X, D)$ as a global quotient. To apply the virtual localization theorem of [GrPa1] we need to verify the technical hypothesis that the moduli space $\overline{\mathcal{M}}_{\Gamma}(X, D)$ admits a $\mathbb{C}^{*}$-equivariant locally closed immersion in a smooth Deligne-Mumford stack (in the case where $(X, D)$ admits a $\mathbb{C}^{*}$-action). In [GrPa1], this is verified for the space of ordinary stable maps to a smooth projective variety with $\mathbb{C}^{*}$ action. The method used there is to realize the moduli space as a quotient stack $[V / G]$ with $V$ a quasi-projective variety and $G$ a reductive group such that the following two conditions are satisfied:

- $V$ is a locally closed subset of a smooth projective variety $W$ such that the $G$-action on $V$ extends to an action on $W$.

- There is a $\left(\mathbb{C}^{*} \times G\right)$-action on $W$ which preserves $V$ and descends to the $\mathbb{C}^{*}$-action on the moduli stack.

We give an analogous construction for the space of relative stable maps. To do this, we use the constructions of [FuPa] and [GrPa1] for stable maps. In [FuPa, Sect. 2], the moduli space of stable maps $\overline{\mathcal{M}}_{g, n}(\mathbb{P}, \beta)$ to a projective space $\mathbb{P}$ (with fixed numerical data $g, n$, $\beta$ ) is expressed as a quotient of a locally closed subscheme $J$ of a Hilbert scheme $H$ (of a product of projective spaces) by a reductive group $G$. As observed in [GrPa1, App. A]:

(i) The stack quotient $[J / G]$ is $\overline{\mathcal{M}}_{g, n}(\mathbb{P}, \beta)$.

(ii) There is a $\left(\mathbb{C}^{*} \times G\right)$-action on $J$ which descends to the given $\mathbb{C}^{*}$-action on $\overline{\mathcal{M}}_{g, n}(\mathbb{P}, \beta)$. 
(iii) There is a $\left(\mathbb{C}^{*} \times G\right)$-equivariant linearized locally closed immersion of $J \subset H$ in a (smooth) Grassmannian $\mathbb{G}$.

Now fix data $\Gamma$ satisfying A1-A4. For any stable relative map in $\overline{\mathcal{M}}_{\Gamma}(X, D)$

$$
f: C \longrightarrow X_{l}
$$

where the target breaks (i.e. $l \geq 1$ ), and any choice of singular locus $D_{i}$ of $X_{l}$, let $\Gamma^{\prime}$ and $\Gamma^{\prime \prime}$ be the "splitting data" of the data $\Gamma$ that arises by separating $X_{l}$ into two pieces. (Thus the stable relative map can be interpreted as a point of $\overline{\mathcal{M}}_{\Gamma^{\prime}}(X, D) \times_{D^{n}} \overline{\mathcal{M}}_{\Gamma^{\prime \prime}}\left(Y, D_{0}, D_{\infty}\right)_{\sim}$.)

Let $M$ be the set of possible splitting data $\left(\Gamma^{\prime}, \Gamma^{\prime \prime}\right)$. Then $M$ is finite, and has a partial ordering defined as follows: if $f: C \rightarrow X_{l}$ is a stable relative map where $l \geq 2$, and breaking the target into $X_{l_{1}} \cup Y_{l-l_{1}}$ yields splitting data $\left(\Gamma_{1}^{\prime}, \Gamma_{1}^{\prime \prime}\right)$, and breaking the target into $X_{l_{2}} \cup Y_{l-l_{2}}$ yields splitting data $\left(\Gamma_{2}^{\prime}, \Gamma_{2}^{\prime \prime}\right)$, and $0 \leq l_{1}<l_{2}<l$, then $\left(\Gamma_{1}^{\prime}, \Gamma_{1}^{\prime \prime}\right) \prec\left(\Gamma_{2}^{\prime}, \Gamma_{2}^{\prime \prime}\right)$. (The reader should verify that this is indeed a partial order.) Extend the partial order to a total order, i.e. choose an order-preserving bijection i : $\{1, \ldots,|M|\} \rightarrow M$.

In $X \times\left(\mathbb{P}^{1}\right)^{|M|}$, define the locus $L_{i}(1 \leq i \leq|M|)$ by $D \times \mathbb{P}^{1} \times \cdots \times\{0\} \times \cdots \times \mathbb{P}^{1}$, where the $\{0\}$ appears in the $i^{\text {th }} \mathbb{P}^{1}$-factor. Blow up $X \times\left(\mathbb{P}^{1}\right)^{|M|}$ along $L_{1}$, then along the proper transform of $L_{2}$, and so on, ending with the proper transform of $L_{|M|}$. Let $\mathrm{Bl}$ denote the result. The singular locus of the morphism $\Phi: \mathrm{Bl} \rightarrow\left(\mathbb{P}^{1}\right)^{|M|}$ is a union of $|M|$ codimension 2 loci, say $\operatorname{Sing}(1), \ldots, \operatorname{Sing}(|M|)$, in order-preserving bijection with $\{1, \ldots,|M|\}$. (In other words, given a fiber of the morphism $\Phi$ isomorphic to $X_{l}$, such that $D_{i_{1}} \subset \operatorname{Sing}\left(j_{1}\right)$ and $D_{i_{2}} \subset \operatorname{Sing}\left(j_{2}\right)$, then $i_{1}<i_{2} \Leftrightarrow j_{1}<j_{2}$.)

There is a $\left(\mathbb{C}^{*}\right)^{1+|M|}$-action on

$$
\left(X \times\left(\mathbb{P}^{1}\right)^{|M|}, L_{1}, \ldots, L_{|M|}\right)
$$

(where the $i^{\text {th }} \mathbb{C}^{*}$ acts on the $i^{\text {th }}$ factor of $X \times\left(\mathbb{P}^{1}\right)^{|M|}$ ) and hence on Bl. Choose a $\left(\mathbb{C}^{*}\right)^{1+|M|_{-}}$ equivariant closed immersion of $\mathrm{Bl}$ into a large projective space $\mathbb{P}$. (As $X$ is a smooth projective variety with $\left(\mathbb{C}^{*}\right)^{1+|M|}$-action, there is a $\left(\mathbb{C}^{*}\right)^{1+|M|}$-equivariant ample invertible sheaf $\mathcal{L}$ on $X$ [MFK, Cor. 1.6, p. 35].)

Then consider the moduli space of stable maps $\overline{\mathcal{M}}_{g, m+n}\left(\mathbb{P}, \beta^{\prime}\right)$, where $\beta^{\prime}$ is the homology class of $\mathbb{P}$ corresponding to the curve mapping to $\beta \in X$. As stated above, this space can be expressed as a quotient satisfying (i)-(iii). Consider the locally closed substack $\overline{\mathcal{M}}^{\prime}$ of $\overline{\mathcal{M}}_{g, m+n}\left(\mathbb{P}, \beta^{\prime}\right)$ corresponding to stable maps:

(I) mapping to $\Phi^{-1}\left(\left(\mathbb{A}^{1}\right)^{|M|}\right) \subset \mathrm{Bl}$;

(II) predeformable as maps to $\mathrm{Bl} \rightarrow\left(\mathbb{P}^{1}\right)^{|M|}$;

(III) for any stable map with image meeting $\operatorname{Sing}(l)$, the splitting type above that singularity is of type $\mathbf{i}(l)$; and

(IV) with the pullback of $D_{\infty}$ given by $\sum \alpha_{i} q_{i}$. 
Then it is straightforward to verify that the quotient $\left[\overline{\mathcal{M}}^{\prime} /\left(\mathbb{C}^{*}\right)^{|M|}\right]$ is isomorphic to $\overline{\mathcal{M}}_{\Gamma}(X, D)$. (For example, from the construction it is clear how to make smooth-local sections of this quotient map.) Note that condition (III) is essential.

Finally, the locally closed immersion $\overline{\mathcal{M}}^{\prime} \hookrightarrow \overline{\mathcal{M}}_{g, m+n}\left(\mathbb{P}, \beta^{\prime}\right)$ lifts to a locally closed immersion $J^{\prime} \hookrightarrow J$, and the $\left(\mathbb{C}^{*}\right)^{|M|}$-action lifts to the Grassmannian $\mathbb{G}$. Hence we have shown the following technical condition required to apply virtual localization.

2.7. Theorem. - The stack $\overline{\mathcal{M}}_{\Gamma}(X, D)$ admits a $\mathbb{C}^{*}$-equivariant closed immersion into a smooth Deligne-Mumford stack.

Specifically, we have shown that conditions (i)-(iii) above hold, with $\overline{\mathcal{M}}_{g, n}(X, \beta)$ replaced by $\overline{\mathcal{M}}_{\Gamma}(X, D) ; J$ replaced by $J^{\prime}$; and $G$ replaced by $G \times\left(\mathbb{C}^{*}\right)^{|M|}$. As a bonus, we observe that $\overline{\mathcal{M}}_{\Gamma}(X, D)$ is a global quotient.

2.8. The perfect obstruction theory. A perfect obstruction theory on $\overline{\mathcal{M}}_{\Gamma}(X, D)$ is constructed and studied in [Li2]. There it is made explicit that this obstruction theory is induced by a relative perfect obstruction theory, relative to the morphism $\overline{\mathcal{M}}_{\Gamma}(X, D) \rightarrow \mathcal{T}$ to the moduli space of targets (Sect. 22.5). In fact, an analysis of the obstruction theory constructed there shows that it is induced by a relative obstruction theory over the product of the (smooth) stacks parametrizing the possible targets and possible sources. That is, if we let $E \rightarrow L_{\overline{\mathcal{M}}_{\Gamma}(X, D)}$ be the perfect obstruction theory on $\overline{\mathcal{M}}_{\Gamma}(X, D)$ and we consider the natural morphism

$$
\Phi: \overline{\mathcal{M}}_{\Gamma}(X, D) \longrightarrow \mathcal{T} \times \mathfrak{M}_{g, m+n},
$$

then there exists an element $F$ in the derived category of sheaves on $\overline{\mathcal{M}}_{\Gamma}(X, D)$ locally representable by a two-term complex of vector bundles, a morphism from $F$ to the relative cotangent complex of the morphism $\Phi$ satisfying the usual cohomological conditions (see [BFn, Sect. 4]), and a distinguished triangle

$$
F[-1] \longrightarrow \Phi^{*} L_{\mathcal{T} \times \mathfrak{M}_{g, m+n}} \longrightarrow E \longrightarrow F
$$

compatible with the morphisms from $E$ and $F$ to the appropriate cotangent complexes.

The reason this is useful is that $F$ can be understood easily in terms of explicit cohomology groups. One should think of the cohomology sheaves of $F$ (or its dual) as measuring the deformations and obstructions of a stable relative map, once deformations of the source and target are chosen. We will explain this carefully here, since this material is spread through a large portion of [Li2] and is not in the form that we need.

We first introduce some notation. If $V$ is a variety, and $D$ is a normal crossings divisor contained in the smooth locus of $V$, then let $\Omega_{V}(\log D)$ be the sheaf of Kähler differentials with logarithmic poles along $D$. Denote the dual of this sheaf by $T_{V}(-\log D)$. When $V$ has normal crossings singularities, this can be interpreted as vector fields on the normalization of $V$ which are tangent to the divisor $D$, tangent to the singular strata, and such that the induced vector fields on the singular strata agree on the different branches 
of the normalization. When $V$ is a nodal curve, $T_{V}(-\log D)$ corresponds to vector fields vanishing at the divisor $D$ and the nodes of $V$.

Finally, if $f: C \rightarrow X_{l} \rightarrow X$ is a relative stable map, then the sheaf $f^{*} T_{X_{l}}\left(-\log D_{\infty}\right)$ will have a torsion subsheaf supported at the distinguished nodes of $C$. This sheaf is the kernel of the morphism from $f^{*} T_{X_{l}}\left(-\log D_{\infty}\right)$ to $\operatorname{Hom}\left(f^{*} \Omega_{X_{l}}\left(\log D_{\infty}, \mathcal{O}_{C}\right)\right.$. We will use the notation $f^{\dagger} T_{X_{l}}\left(-\log D_{\infty}\right)$ to denote the quotient of $f^{*} T_{X_{l}}\left(-\log D_{\infty}\right)$ by this subsheaf. Sections of $f^{\dagger} T_{X_{l}}\left(-\log D_{\infty}\right)$ when restricted to an irreducible component $C^{\prime}$ of $C$ correspond simply to sections of the pullback of the restriction of the logarithmic tangent bundle to the irreducible component of $X_{l}$ to which $C^{\prime}$ maps. At a distinguished node, the sections on the two components of $C$ are required to agree in the sense that they give the same element of the tangent space to $D$.

If $C \stackrel{f}{\rightarrow} X_{l} \rightarrow X$ is a stable relative map to $(X, D)$, then it is easy to compute that the $\Phi$-relative tangent space to the moduli space at this point is

$$
\operatorname{RelDef}(f)=H^{0}\left(C, f^{\dagger} T_{X_{l}}\left(-\log D_{\infty}\right)\right) .
$$

It is important not to confuse this space with the space $\operatorname{Hom}\left(f^{*} \Omega_{X_{l}}\left(\log D_{\infty}\right), \mathcal{O}_{C}\right)$ which parametrizes deformations of the map preserving the contact conditions along $D_{\infty}$, but ignoring the predeformability condition at the nodes. The space of relative obstructions $\operatorname{RelOb}(f)$ has a natural filtration

$$
0 \rightarrow H^{1}\left(C, f^{*} T_{X_{l}}\left(-\log D_{\infty}\right)\right) \rightarrow \operatorname{RelOb}(f) \rightarrow H^{0}\left(C, f^{-1} \mathcal{E x t}^{1}\left(\Omega_{X_{l}}\left(\log D_{\infty}\right), \mathcal{O}_{X_{l}}\right)\right) \rightarrow 0
$$

Because $D_{\infty}$ is contained in the smooth locus of $X_{l}$, there is a natural isomorphism between $\mathcal{E} \mathrm{xt}^{1}\left(\Omega_{X_{l}}\left(\log D_{\infty}\right), \mathcal{O}_{X_{l}}\right)$ and $\mathcal{E x t}^{1}\left(\Omega_{X_{l}}, \mathcal{O}_{X_{l}}\right)$. We can make this sheaf more explicit as follows. $\mathcal{E x t}^{1}\left(\Omega_{X_{l}}, \mathcal{O}_{X_{l}}\right)$ is the pushforward of a trivial line bundle on $\operatorname{Sing} X_{l}=\cup_{i=0}^{l-1} D_{i}$. However, there is no canonical trivialization of this line bundle, and in families it can vary. On $D_{i}$ this line bundle is canonically isomorphic to $N_{D_{i} / X(i-1)} \otimes N_{D_{i} / X(i)}$, where $X(i-1)$ and $X(i)$ are the components of $X_{l}$ containing $D_{i}$ (we will not use this notation $X(i)$ again).

Sequence (2) is a close analog of the local-to-global sequence for Ext and has the same deformation-theoretic interpretation: the right-hand group represents the local obstructions to deforming $f$, and the left-hand group is the global obstruction. We now explain explicitly why these groups are as above.

For each singular locus $D_{i}$ of $X_{l}$, we denote the set of nodes of $C$ mapping to $D_{i}$ by $\left\{N_{i}^{1}, \ldots, N_{i}^{j_{i}}\right\}$. We can rewrite sequence (2) as

$$
0 \longrightarrow H^{1}\left(C, f^{\dagger} T_{X_{l}}\left(-\log D_{\infty}\right)\right) \longrightarrow \operatorname{RelOb}(f) \longrightarrow \bigoplus_{i=0}^{l-1} L_{i}^{\oplus j_{i}} \longrightarrow 0
$$

where $L_{i}$ is the one-dimensional vector space of sections of the sheaf $\left.\mathcal{E x t}^{1}\left(\Omega_{X_{l}}, \mathcal{O}_{X_{l}}\right)\right|_{D_{i}}$, which can be interpreted as the deformation space of the singularity $D_{i}$ of $X_{l}$. The last term is the local obstruction to extending a map, which comes from a compatibility requirement between the choices of deformation of a singularity $D_{i}$ of the target and the deformation of those nodes of the source $N_{i}^{j}$ which map to $D_{i}$. The fact that this obstruction space is identified with the deformation space of the target singularity $D_{i}$ can be seen from a direct local calculation. 
Explicitly, suppose we have a predeformable morphism between nodal curves over a base Spec $R$ with $R$ an Artin local ring with maximal ideal $\mathfrak{m}$. For notational convenience (to avoid the irrelevant variables $z_{i}$ of Sect. 2.3) we assume $\operatorname{dim} X=1$. Étale-locally, the predeformable morphism has the form $C \rightarrow X_{l}$ with $X_{l}=\operatorname{Spec}(R[x, y] /(x y-a))$ and $C=\operatorname{Spec}(R[u, v] /(u v-b))$ with $a, b \in \mathfrak{m}$ and the morphism is given by $x \mapsto \alpha u^{n}, y \mapsto \beta v^{n}$. If $\tilde{R}$ is a small extension of $R$ with ideal $I$, then choosing lifts of $C$ and $X_{l}$ to $\operatorname{Spec} \tilde{R}$ corresponds to lifting the elements $a$ and $b$ in $R$ to elements $\tilde{a}$ and $\tilde{b}$ in $\tilde{R}$. These choices are torsors for the ideal $I$, but the structure of $I$-torsor depends on the choice of local coordinates on $C$ and $X_{l}$. The choice of $\tilde{a}$ is canonically a torsor for $I \otimes L_{i}$ where $L_{i}$ is the one-dimensional deformation space of the singularity $D_{i}$ of $X_{l}$.

If we want the morphism to extend, then the choice of $\tilde{b}$ determines the choice of $\tilde{a}$, because we have the formula $a=x y=\alpha \beta u^{n} v^{n}=\alpha \beta b^{n}$. If we choose a lifting $\tilde{b}$ of $b$ and then try to choose liftings $\tilde{\alpha}, \tilde{\beta}, \tilde{a}$ satisfying the analogous formula, we see that $\tilde{a}$ is determined, since the ambiguity in the choice of $\tilde{\alpha}$ and $\tilde{\beta}$ is an element of $I$ which is annihilated by multiplication with $\tilde{b}$. Hence, given choices of $\tilde{b}$ and $\tilde{a}$, the element $\tilde{a}-\alpha \beta \tilde{b}^{n}$ is a canonically defined element of $I \otimes L_{i}$ which vanishes exactly if there is a local extension of the predeformable morphism.

The first term in Sequence (2) is the global obstruction, analogous to the term $H^{1}\left(C, f^{*} T_{X}\right)$ in the case of ordinary stable maps, which can be identified by the usual Čech cocycle construction once the local obstructions vanish. Explicitly, suppose we are given a morphism $C \rightarrow X_{l}$ over Spec $R$ and liftings $\tilde{C}$ and $\tilde{X}_{l}$ as above. Then if all the local obstructions constructed above vanish, we can étale-locally extend the morphism. That is, we can choose an étale cover of $C$ by open sets $U_{i}$ inducing an étale cover of $\tilde{C}$ by open sets $\tilde{U}_{i}$, and on each $\tilde{U}_{i}$ there exists an extension of the morphism. The set of such extensions is a $H^{0}\left(U_{i}, f^{\dagger} T_{X_{l}}(-\log D)\right)$-torsor, so taking the differences on overlaps we get a Čech cocycle representing an element of $H^{1}\left(C, f^{\dagger} T_{X_{l}}(-\log D)\right)$ which gives the global obstruction. (Here we use the fact that étale cohomology agrees with Zariski cohomology for coherent sheaves.)

2.9. The perfect obstruction theory of $\overline{\mathcal{M}}_{\Gamma}\left(Y, D_{0}, D_{\infty}\right) \sim$. The relative obstruction theory for $\overline{\mathcal{M}}_{\Gamma}\left(Y, D_{0}, D_{\infty}\right)_{\sim}$ (over the moduli stack of source and target) is identical to the previous (rigid) case, with $\mathcal{T}$ replaced by $\mathcal{T}_{\sim}$.

\section{RELATIVE VIRTUAL LOCALIZATION}

In [GrPa1], a localization formula is proved for the virtual fundamental class in the general context of $\mathbb{C}^{*}$-equivariant perfect obstruction theories. If $\mathcal{M}$ is a Deligne-Mumford stack with such an obstruction theory, then the $\mathbb{C}^{*}$-fixed loci $\mathcal{M}_{i}$ carry an associated $\left(\mathbb{C}^{*}\right.$ fixed) perfect obstruction theory, giving them each a virtual fundamental class. The virtual normal bundle to $\mathcal{M}_{i}$ arises from the "moving" (non-torus-fixed) part of the obstruction theory. Assume that $\mathcal{M}$ has an equivariant locally closed immersion into a smooth 
Deligne-Mumford stack. The virtual localization formula [GrPa1, equ. (1)] then states:

$$
[\mathcal{M}]^{\mathrm{vir}}=\sum \frac{\left[\mathcal{M}_{i}\right]^{\mathrm{vir}}}{e\left(N_{i}^{\mathrm{vir}}\right)}
$$

in $A_{*}^{\mathbb{C}^{*}}(\mathcal{M}) \otimes \mathbb{Q}\left[t, \frac{1}{t}\right]$, where $t$ is the generator of the $\mathbb{C}^{*}$-equivariant Chow ring of a point. (In applications, the equation is usually capped with the Chow classes of equivariant vector bundles to obtain equalities of intersection numbers. Our strategy is to cap with classes of lower codimension, to prove equalities in the Chow ring.)

The primary example there was the moduli space of stable maps to $\mathbb{P}^{n}$ [GrPa1, Sect. 4]. In that case the virtual normal bundle is given explicitly by contributions from parts of the geometry of the maps in $\mathcal{M}_{i}$.

In this section, we suppose $X$ is a smooth projective variety with a non-trivial $\mathbb{C}^{*}$ action, and $D$ is an irreducible divisor in the fixed locus. (The arguments below apply essentially without change in greater generality, for example when $D$ is reducible or the group is larger, but for the sake of exposition we will state relative virtual localization in only moderate generality.) The natural $\mathbb{C}^{*}$-action on the perfect obstruction theory of $\overline{\mathcal{M}}_{\Gamma}(X, D)$ gives this space an equivariant virtual fundamental class.

3.1. $\mathbb{C}^{*}$-fixed loci in the relative setting, and their virtual fundamental classes. We now set notation for the $\mathbb{C}^{*}$-fixed locus of $\overline{\mathcal{M}}_{\Gamma}(X, D)$. If a connected component of the fixed locus has general morphism with target $X$ (i.e. the target doesn't degenerate for the general morphism, or equivalently for any morphism in this connected component of the fixed locus), we say that it is a simple fixed locus. Otherwise, it is a composite fixed locus.

A simple fixed locus has an induced obstruction theory that is the fixed part of the obstruction theory of $\overline{\mathcal{M}}_{\Gamma}(X, D)$, and hence a virtual fundamental class, and a virtual normal bundle, which we denote $N_{\Gamma}$. The analysis of the perfect obstruction theory here is much simpler than the general case, because the terms corresponding to deformations and automorphisms of the target as well as the term giving the local obstruction to deforming the morphism vanish. Thus the analysis of the virtual normal bundle to such a component is identical to the case of ordinary stable maps with the bundle $T_{X}$ systematically replaced by $T_{X}(-\log D)$. Denote the union of simple fixed loci by $\overline{\mathcal{M}}_{\Gamma}(X, D)^{\text {simple }}$.

Any element of a composite fixed locus is of the form $f: C^{\prime} \cup C^{\prime \prime} \rightarrow X_{l}(l>0)$, which restricts to $f^{\prime}: C^{\prime} \rightarrow X$ and $f^{\prime \prime}: C^{\prime \prime} \rightarrow Y_{l}$ which agree over the nodes $\left\{N_{1}, \ldots, N_{\delta}\right\}=C^{\prime} \cap$ $C^{\prime \prime}$. Let $\Gamma^{\prime}$ be the data A1-A4 corresponding to $f^{\prime}$, and $\Gamma^{\prime \prime}$ be the data B1-B4 corresponding to $f^{\prime \prime}$. (Any two of $\left\{\Gamma, \Gamma^{\prime}, \Gamma^{\prime \prime}\right\}$ determine the third.) Define $m_{i}$ by $\left(f^{\prime}\right)^{-1}(D)=\sum m_{i} N_{i}$ on $C^{\prime}$, or equivalently $\left(f^{\prime \prime}\right)^{-1}\left(D_{0}\right)=\sum m_{i} N_{i}$ on $C^{\prime \prime}$; this is part of the data of both $\Gamma^{\prime}$ and $\Gamma^{\prime \prime}$. Both $\Gamma^{\prime}$ and $\Gamma^{\prime \prime}$ are locally constant on the fixed locus.

The fixed locus $\overline{\mathcal{F}}_{\Gamma^{\prime}, \Gamma^{\prime \prime}}$ corresponding to a given $\Gamma^{\prime}$ and $\Gamma^{\prime \prime}$ is canonically the (étale) quotient of the stack

$$
\overline{\mathcal{M}}_{\Gamma^{\prime}, \Gamma^{\prime \prime}}=\overline{\mathcal{M}}_{\Gamma^{\prime}}(X, D)^{\text {simple }} \times_{D^{n}} \overline{\mathcal{M}}_{\Gamma^{\prime \prime}}\left(Y, D_{0}, D_{\infty}\right)_{\sim},
$$


by the finite group $\operatorname{Aut}(m$. $)$ (those permutations of $(1, \ldots, \delta)$ preserving $\left.\left(m_{1} \ldots, m_{\delta}\right)\right)$. Call this quotient morphism $g l$, so

$$
g l: \overline{\mathcal{M}}_{\Gamma^{\prime}, \Gamma^{\prime \prime}} \rightarrow \overline{\mathcal{F}}_{\Gamma^{\prime}, \Gamma^{\prime \prime}}
$$

(Cf. [Li1, Prop. 4.13]; the morphism $g l$ is called $\Phi$ there.)

A virtual fundamental class on $\overline{\mathcal{M}}_{\Gamma^{\prime}, \Gamma^{\prime \prime}}$ (and $\left.\overline{\mathcal{F}}_{\Gamma^{\prime}, \Gamma^{\prime \prime}}\right)$, which we term the glued fundamental class, is induced by the virtual fundamental classes on the factors (cf. [Li2, p. 203]):

$$
\begin{aligned}
{\left[\overline{\mathcal{M}}_{\Gamma^{\prime}, \Gamma^{\prime \prime}}\right]^{\text {glued }} } & =\Delta^{!}\left(\left[\overline{\mathcal{M}}_{\Gamma^{\prime}}(X, D)^{\text {simple }}\right]^{\text {vir }} \times\left[\overline{\mathcal{M}}_{\Gamma^{\prime \prime}}\left(Y, D_{0}, D_{\infty}\right)_{\sim}\right]^{\text {vir }}\right) \\
{\left[\overline{\mathcal{F}}_{\Gamma^{\prime}, \Gamma^{\prime \prime}}\right]^{\text {glued }} } & =\frac{1}{\mid \text { Aut }(m .) \mid} g l_{*}\left[\overline{\mathcal{M}}_{\Gamma^{\prime}, \Gamma^{\prime \prime}}\right]^{\text {glued }}
\end{aligned}
$$

where $\Delta: D^{n} \rightarrow D^{n} \times D^{n}$ is the diagonal morphism. A second virtual fundamental class on $\overline{\mathcal{F}}_{\Gamma^{\prime}, \Gamma^{\prime \prime}}$ (and by pullback, on $\left.\overline{\mathcal{M}}_{\Gamma^{\prime}, \Gamma^{\prime \prime}}\right),\left[\overline{\mathcal{F}}_{\Gamma^{\prime}, \Gamma^{\prime \prime}}\right]^{\mathrm{vir}}\left(\right.$ resp. $\left[\overline{\mathcal{M}}_{\Gamma^{\prime}, \Gamma^{\prime \prime}}\right]^{\mathrm{vir}}$ ), is that induced by the $\mathbb{C}^{*}$-fixed part of the pullback of the obstruction theory of $\overline{\mathcal{M}}_{\Gamma}(X, D)$. The following lemma shows that they are the same.

3.2. Lemma. - The torus-induced virtual fundamental class on a composite fixed locus is exactly the glued virtual fundamental class coming from the two factors. In other words,

$$
\left[\overline{\mathcal{F}}_{\Gamma^{\prime}, \Gamma^{\prime \prime}}\right]^{\text {glued }}=\left[\overline{\mathcal{F}}_{\Gamma^{\prime}, \Gamma^{\prime \prime}}\right]^{\text {vir }} \quad \text { and } \quad\left[\overline{\mathcal{M}}_{\Gamma^{\prime}, \Gamma^{\prime \prime}}\right]^{\text {glued }}=\left[\overline{\mathcal{M}}_{\Gamma^{\prime}, \Gamma^{\prime \prime}}\right]^{\text {vir }} \text {. }
$$

Proof. We relate the obstruction theory for $f$ to the obstruction theories for $f^{\prime}$ and $f^{\prime \prime}$ separately. We use the description of the obstruction theory given in Section 2.9. A priori, these virtual classes are defined relative to two different bases. The first is defined relative to $\mathcal{T} \times \mathfrak{M}_{g, m+n}$ and the second is defined relative to $\mathcal{T} \times \mathcal{T}_{\sim} \times \mathfrak{M}_{g^{\prime}, m^{\prime}+\delta} \times \mathfrak{M}_{g^{\prime \prime}, m^{\prime \prime}+\delta+n}$. However, the torus action on the relative cotangent complex of the map between these two base spaces has no torus fixed part, because the $\mathbb{C}^{*}$-action on the deformation space of $D_{0}$ is nontrivial, as is the torus action on the deformation spaces of the $\delta$ nodes $\left\{N_{i}\right\}$. Therefore we need only consider the relative obstruction theories. By (1) and (2) there are two pieces here: one is $H^{\cdot}\left(C, f^{\dagger} T_{X}(-\log D)\right)$, and the other is the local obstruction at the nodes mapping to the singular locus of $X_{l}$. The local obstruction coming from the node $N_{i}$ has a nontrivial $\mathbb{C}^{*}$-action, because the weight of this torus action is the same as the weight of the action on $N_{D / X}$. Thus, they do not contribute to the fixed obstruction theory. The local obstructions coming from the singular locus of $Y_{l}$ have zero weight, since the torus doesn't act on $Y_{l}$, meaning that these local obstructions occur in the torus-fixed part of the perfect obstruction theory, just as they do in the obstruction theory on $\overline{\mathcal{M}}_{\Gamma^{\prime \prime}}$. There are no local obstructions for $f^{\prime}$, since the target is smooth. We study the global obstructions using the partial normalization map $C^{\prime} \amalg C^{\prime \prime} \rightarrow C$. This gives us a long exact sequence in cohomology

$$
\begin{gathered}
0 \longrightarrow H^{0}\left(C, f^{\dagger} T_{X_{l}}\left(-\log D_{\infty}\right)\right) \\
\longrightarrow H^{0}\left(C^{\prime}, f^{\prime *}\left(T_{X}(-\log D)\right)\right) \oplus H^{0}\left(C^{\prime \prime}, f^{\prime \prime \dagger}\left(T_{Y_{l}}\left(-\log D_{0}-\log D_{\infty}\right)\right)\right) \\
\left.\longrightarrow \oplus_{i=1}^{n} T_{D}\right|_{f\left(N_{i}\right)} \longrightarrow H^{1}\left(C, f^{\dagger}\left(T_{X_{l}}\left(-\log D_{\infty}\right)\right)\right) \\
\longrightarrow H^{1}\left(C^{\prime}, f^{\prime *}\left(T_{X}(-\log D)\right)\right) \oplus H^{1}\left(C^{\prime \prime}, f^{\dagger}\left(T_{Y_{l}}\left(-\log D_{0}-\log D_{\infty}\right)\right)\right) \longrightarrow 0 .
\end{gathered}
$$


Note that this looks slightly different from the standard normalization sequences, because the agreement required at the nodes is only in the space $T_{D}$. The torus action on $T_{D}$ is trivial, since the divisor is torus-fixed. We conclude that the difference between the zero-weight piece of this obstruction theory and the zero-weight piece of the obstruction theory coming from $f^{\prime}$ and $f^{\prime \prime}$ separately is precisely the term $\left.\oplus T_{D}\right|_{f\left(N_{i}\right)}$. This is simply the pullback of the normal bundle of the diagonal morphism from $D^{n}$ to $D^{2 n}$ (which corresponds to the refined Gysin homomorphism $\Delta^{!}$in (4)). Thus, this sequence is exactly the verification of the compatibility condition of perfect obstruction theories that guarantees that the two possible choices of virtual class on this moduli space agree (see for example [BFn, Prop. 5.10]). (This is essentially the same as the standard argument used to prove the splitting axiom in Gromov-Witten theory.)

3.3. Two virtual bundles on $\overline{\mathcal{M}}_{\Gamma^{\prime}, \Gamma^{\prime \prime}}$ and $\overline{\mathcal{F}}_{\Gamma^{\prime}, \Gamma^{\prime \prime}}$. We next describe two relevant (virtual) bundles on $\overline{\mathcal{M}}_{\Gamma^{\prime}, \Gamma^{\prime \prime}}$ and $\overline{\mathcal{F}}_{\Gamma^{\prime}, \Gamma^{\prime \prime}}$. First, the pullback of $N_{\Gamma^{\prime}}$ to $\overline{\mathcal{M}}_{\Gamma^{\prime}, \Gamma^{\prime \prime}}$ is a virtual bundle which we will also denote by $N_{\Gamma^{\prime}}$. This bundle naturally descends to $\overline{\mathcal{F}}_{\Gamma^{\prime}, \Gamma^{\prime \prime}}$. For convenience, we denote the descended bundle $N_{\Gamma^{\prime}}$ as well.

The second relevant bundle is the line bundle $\mathcal{L}$ corresponding to the deformation of the singularity $D_{0}\left(=X \cap Y_{l}\right.$ in $X_{l}$ ). (This line bundle $\mathcal{L}$ is analogous to the line bundle 1 arising in J. Li's degeneration formula [Li2, p. 203].) The fiber of this bundle at a point of the boundary is canonically isomorphic to $H^{0}\left(D, N_{D / X} \otimes N_{D_{0} / Y_{l}}\right)$. The line bundle $N_{D / X} \otimes N_{D_{0} / Y_{l}}$ is trivial on $D$, so its space of global sections is one-dimensional, and we can canonically identify this space of sections with the fiber of the line bundle at any point $p t$ of $D$. Thus we see that we can write the bundle $\mathcal{L}$ as a tensor product of bundles pulled back from the two factors separately. The one coming from $\overline{\mathcal{M}}_{\Gamma^{\prime}}(X, D)$ is trivial, since it is globally identified with $H^{0}\left(p t,\left.N_{D / X}\right|_{p t}\right)$, but it has a nontrivial torus action; we denote this weight (i.e. the first Chern class of this bundle, which is pure weight) by $w$. In other words, $w$ is the weight of the torus action on the normal bundle to $D$ in $X$. The line bundle coming from $\overline{\mathcal{M}}_{\Gamma^{\prime \prime}}(Y)$ is a nontrivial line bundle, but with trivial torus action; this is precisely the pullback of $-\psi$ (where $\psi$ was defined in Section 2.5). Thus

$$
c_{1}(\mathcal{L})=w-\psi
$$

3.4. Remark. For each node $N_{i}$ joining $C^{\prime}$ to $C^{\prime \prime}$, there is a natural isomorphism between the line bundle $\mathcal{L}$ and the $m_{i}^{\text {th }}$ tensor power of the line bundle corresponding to the deformation of the singularity of $C$ at $N_{i}$. This is because the morphism from $C^{\prime}$ (respectively $\left.C^{\prime \prime}\right)$ induces an isomorphism between $\left.T_{C^{\prime}}^{\otimes m_{i}}\right|_{N_{i}}$ and $\left.N_{D / X}\right|_{f\left(N_{i}\right)}$ (respectively $\left.T_{C^{\prime \prime}}^{\otimes m_{i}}\right|_{N_{i}}$ and $\left.\left.N_{D_{0} / Y}\right|_{f\left(N_{i}\right)}\right)$.

3.5. We may now state the relative virtual localization theorem, which reduces understanding the contributions of an arbitrary fixed locus to understanding the contributions from a simple fixed locus and the contributions from maps to a non-rigid target. 
3.6. Theorem (Relative virtual localization). -

$$
\begin{aligned}
{\left[\overline{\mathcal{M}}_{\Gamma}(X, D)\right]^{\text {vir }} } & =\frac{\left[\overline{\mathcal{M}}_{\Gamma}(X, D)^{\text {simple }}\right]^{\text {vir }}}{e\left(N_{\Gamma}\right)}+\sum_{\overline{\mathcal{M}}_{\Gamma^{\prime}, \Gamma^{\prime \prime}} \text { composite }}\left(\prod m_{i}\right) \frac{\left[\overline{\mathcal{F}}_{\Gamma^{\prime}, \Gamma^{\prime \prime}}\right]^{\text {vir }}}{e\left(N_{\Gamma^{\prime}}\right) c_{1}(\mathcal{L})} \\
& =\frac{\left[\overline{\mathcal{M}}_{\Gamma}(X, D)^{\text {simple }}\right]^{\text {vir }}}{e\left(N_{\Gamma}\right)}+\sum_{\overline{\mathcal{M}}_{\Gamma^{\prime}, \Gamma^{\prime \prime}} \text { composite }} \frac{\left(\prod m_{i}\right) g l_{*}\left[\overline{\mathcal{M}}_{\Gamma^{\prime}, \Gamma^{\prime \prime}}\right]^{\text {glued }}}{|\operatorname{Aut}(m .)| e\left(N_{\Gamma^{\prime}}\right)(w-\psi)}
\end{aligned}
$$

The two versions of the theorem are obviously equivalent by Lemma 3.2 Relative virtual localization may be interpreted (and proven) as follows. First, the induced virtual fundamental class on the fixed locus agrees with the natural virtual fundamental class coming from the modular interpretation of the fixed locus (Lemma 3.2). Second, there is a contribution to the virtual normal bundle not present on the moduli space of stable maps which arises from the deformation of the target in the case of composite fixed loci. This contribution is virtual codimension 1 (in a more precise sense virtual codimension $(\delta+1)-\delta$, as we shall see in the proof), and the contribution to the Euler class of the virtual normal bundle is $c_{1}(\mathcal{L}) / \prod m_{i}$.

3.7. Corollary: Relative virtual localization with target $\mathbb{P}^{1}$. To prove Theorem $\star$, we will use relative virtual localization for target $\mathbb{P}^{1}$. In this case we geometrically interpret $N_{\Gamma^{\prime}}$ by giving the changes required from the formula of [GrPa1, p. 505]. We choose the $\mathbb{C}^{*}$-action on $\mathbb{P}^{1}$ that acts with weight 1 on $T_{\mathbb{P}^{1}}$ at 0 and weight -1 at $\infty$; for example, we can take $\lambda_{0}=t$ and $\lambda_{\infty}=0$ in the language of [GrPa1] (where $t$ is the generator of equivariant Chow ring of a point). Thus $w=-t$.

Then the formula for $e\left(N_{\Gamma^{\prime}}\right)$ is the same as the formula for $e\left(N^{\mathrm{vir}}\right)$ of [GrPa1, p. 505] except $\lambda_{0}=t$ and $\lambda_{\infty}=0$, and the edge contribution should be

$$
\prod_{\text {edges } e} \frac{d_{e}^{d_{e}}}{d_{e} ! t^{d_{e}}} .
$$

This accounts for replacing $T_{\mathbb{P}^{1}}$ with $T_{\mathbb{P}^{1}}(-\infty)$. There is no correction to the vertex contributions: because there are no nodes or contracted components over infinity, the other terms that could conceivably change are unaffected by this replacement of bundles.

We now give two proofs of the Relative virtual localization theorem 3.6. The first is short and direct, using the description of the perfect obstruction theory given in Section 2.9. The second proof avoids the technical details of the perfect obstruction theory by invoking more results from [Li2] and independently establishes the equality of virtual classes of Lemma 3.2. (This is possible because the technical machinery required for relative virtual localization is very similar to that needed for Li's degeneration formula.)

3.8. The first proof of relative virtual localization. To prove our relative virtual localization statement, what remains is to analyze the virtual normal bundle, which is determined from the non-fixed part of the perfect obstruction theory. Part of this virtual bundle is just the virtual normal bundle of the locus $\overline{\mathcal{M}}_{\Gamma^{\prime}}$ inside $\overline{\mathcal{M}}_{\Gamma^{\prime}}(X, D)$. (The $\mathbb{C}^{*}$-action on $\overline{\mathcal{M}}_{\Gamma^{\prime \prime}}\left(Y, D_{0}, D_{\infty}\right)_{\sim}$ is trivial, so there is no virtual normal bundle on that side.) All that 
remains are the terms coming from the nodes $\left\{N_{i}\right\}$ of $C$ and the singularities of $X_{l}$. There is a single deformation of $X_{l}$ with nonzero torus weight corresponding to smoothing the singularity $D_{0}$ of $X_{l}$. This contributes a copy of $\mathcal{L}$ to the virtual normal bundle. For each of the $N_{i}$ there is a deformation of $C$ corresponding to smoothing the node at $N_{i}$, giving a contribution of $\frac{1}{m_{i}} \mathcal{L}$ (Remark 3.4). Finally, each $N_{i}$ also contributes a local obstruction which we have seen is given by $\mathcal{L}\left(\right.$ see $(3)$ ). We cancel the resulting copy of $c_{1}(\mathcal{L})$ that occurs in both the numerator and denominator $n$ times, leaving $\frac{1}{\prod m_{i}}$. We conclude that the difference between the Euler class of the virtual normal bundle to $\overline{\mathcal{M}}_{\Gamma^{\prime}, \Gamma^{\prime \prime}}$ in $\overline{\mathcal{M}}_{\Gamma}(X, D)$ and the Euler class of the pullback of the virtual normal bundle of $\overline{\mathcal{M}}_{\Gamma^{\prime}}$ in $\overline{\mathcal{M}}_{\Gamma^{\prime}}(X, D)$ is given by $\frac{1}{\prod m_{i}} c_{1}(\mathcal{L})$, which completes the proof of Theorem 3.6

3.9. The second proof of relative virtual localization. We again show that the Euler class of the virtual normal bundle to $\overline{\mathcal{F}}_{\Gamma^{\prime}, \Gamma^{\prime \prime}}$ is $e\left(N_{\Gamma^{\prime}}\right)(w-\psi) / \prod m_{i}$. We first define two virtual divisors on $\overline{\mathcal{M}}_{\Gamma}(X, D)$, denoted $Z_{\text {thick }}$ and $Z_{\text {thin }}$.

There is a line bundle $\mathcal{L}^{\prime}$ with section $s_{\mathcal{L}^{\prime}}$ on $\overline{\mathcal{M}}_{\Gamma}(X, D)$ corresponding to the locus where the target breaks, and the source curve data splits into $\Gamma^{\prime}$ and $\Gamma^{\prime \prime}$. Let $Z_{\text {thick }}$ be the virtual Cartier divisor corresponding to $s_{\mathcal{L}^{\prime}}$. Étale-locally near a point of $Z_{\text {thick, }}$ the divisor is pulled back from the deformation space of the singularity $D_{\Gamma^{\prime}, \Gamma^{\prime \prime}}$ of $X_{l}$ splitting the source curve data into $\Gamma^{\prime}$ and $\Gamma^{\prime \prime}$. Then $Z_{\text {thick }}$ has an induced obstruction theory, and hence a virtual fundamental class given by

$$
\left[Z_{\text {thick }}\right]^{\text {vir }}=c_{1}\left(\mathcal{L}^{\prime}\right) \cap\left[\overline{\mathcal{M}}_{\Gamma}(X, D)\right]^{\text {vir }}
$$

by [Li2, Lemma 3.11] (see also [Li2, Lemma 4.6]).

The restriction of $\mathcal{L}^{\prime}$ to $\overline{\mathcal{F}}_{\Gamma^{\prime}, \Gamma^{\prime \prime}}$ is the line bundle $\mathcal{L}$ of Section 3.3. (Caution: Although $c_{1}(\mathcal{L})=w-\psi, c_{1}\left(\mathcal{L}^{\prime}\right) \neq w-\psi$ on $\overline{\mathcal{M}}_{\Gamma}(X, D)$ in general, because $Z_{\text {thick }}$ may contain maps corresponding to points $\overline{\mathcal{M}}_{\Gamma^{\prime}}\left(X, D_{\Gamma^{\prime}, \Gamma^{\prime \prime}}\right)$ where the target $X$ degenerates. Then the normal bundle $N$ to the $D_{\Gamma^{\prime}, \Gamma^{\prime \prime}}$ in the degeneration of $X$ is not the same equivariantly as $N_{D / X}$.)

Define $Z_{\text {thin }}$ as the closed substack of $Z_{\text {thick }}$ where we require furthermore that the nodes of the source curve mapping to $D_{\Gamma^{\prime}, \Gamma^{\prime \prime}}$ are not smoothed (even to first order):

$$
Z_{\text {thin }}=g l\left(\overline{\mathcal{M}}_{\Gamma^{\prime}}(X, D) \times_{D^{n}} \overline{\mathcal{M}}_{\Gamma^{\prime \prime}}\left(Y, D_{0}, D_{\infty}\right)_{\sim}\right)
$$

where $g l$ again is the étale quotient map by the finite group Aut $\left(m\right.$.). $Z_{\text {thin }}$ is given an obstruction theory in [Li2, p. 250-252]. Let $\left[Z_{\text {thin }}\right]^{\text {vir }}$ be the associated virtual fundamental class. A second virtual fundamental class (and obstruction theory) comes from the gluing description:

$$
\left[Z_{\text {thin }}\right]^{\text {glued }}=\frac{1}{|\operatorname{Aut}(m .)|} g l_{*} \Delta^{!}\left(\left[\overline{\mathcal{M}}_{\Gamma^{\prime}}(X, D)\right]^{\text {vir }} \times_{D^{n}}\left[\overline{\mathcal{M}}_{\Gamma^{\prime \prime}}\left(Y, D_{0}, D_{\infty}\right)_{\sim}\right]^{\text {vir }}\right)
$$

(compare to (4)). By [Li2, Lemma 3.14], $\left[Z_{\text {thin }}\right]^{\text {glued }}=\left[Z_{\text {thin }}\right]^{\text {vir }}$, and in fact the two obstruction theories are identical [Li2, Lemma 4.15].

Consider the sequence of inclusions

$$
\overline{\mathcal{F}}_{\Gamma^{\prime}, \Gamma^{\prime \prime}} \subset Z_{\text {thin }} \subset Z_{\text {thick }} \subset \overline{\mathcal{M}}_{\Gamma}(X, D) .
$$


(The last three terms should be compared to the first, second, and fourth terms of the sequence of inclusions of [Li2, p. 248].) For each inclusion, we identify the virtual normal bundle of each term in the next, and verify that it has no fixed part when restricted to $\overline{\mathcal{F}}_{\Gamma^{\prime}, \Gamma^{\prime \prime}}$. As the obstruction theory of $\overline{\mathcal{F}}_{\Gamma^{\prime}, \Gamma^{\prime \prime}}$ is $\mathbb{C}^{*}$-fixed, it follows that the obstruction theory of $\overline{\mathcal{F}}_{\Gamma^{\prime}, \Gamma^{\prime \prime}}$ is indeed the fixed part of the restriction of the obstruction theory of $\overline{\mathcal{M}}_{\Gamma}(X, D)$. By multiplying the Euler classes of the virtual normal bundles of each inclusion, we obtain the Euler class of the virtual normal bundle of $\overline{\mathcal{F}}_{\Gamma^{\prime}, \Gamma^{\prime \prime}}$, completing the proof of relative virtual localization.

The inclusion $Z_{\text {thick }} \subset \overline{\mathcal{M}}_{\Gamma}(X, D)$. As stated earlier, the virtual normal bundle of $Z_{\text {thick }}$ in $\overline{\mathcal{M}}_{\Gamma}(X, D)$ is $\mathcal{L}^{\prime}$. When restricted to $\overline{\mathcal{F}}_{\Gamma^{\prime}, \Gamma^{\prime \prime}}$, it is $\mathcal{L}$, which has no fixed part, as $\mathbb{C}^{*}$ acts on $\mathcal{L}$ nontrivially (by our assumption that $\mathbb{C}^{*}$ does not act trivially on $X$ ).

The inclusion $Z_{\text {thin }} \subset Z_{\text {thick. }}$. We need to delve into Li's argument of [Li2, Sect. 4.4], where the obstruction theories of $Z_{\text {thin }}$ and $Z_{\text {thick }}$ are compared, to verify that the fixed parts of both obstruction theories (when restricted to $\overline{\mathcal{F}}_{\Gamma^{\prime}, \Gamma^{\prime \prime}}$ ) are the same. We refer the reader in particular to the first paragraph of that section for an overview of the strategy.

We motivate the argument with a simple example. Consider the morphism of schemes from the formal neighborhood of $\delta$ nodes to the formal neighborhood of a node, where the morphism is predeformable, with the $i^{\text {th }}$ node of the source mapping with ramification $m_{i}$. The deformation space of this morphism is reducible if $\prod m_{i}>1$ : if (to first order) any of the branchings of the source curve move away from the (scheme-theoretic) preimage of the target node, then the target node cannot smooth, even to first order. There is precisely one component that surjects onto the deformation space of the target node. Formal equations for this component are

$$
\operatorname{Spf} \mathbb{C}\left[\left[y_{1}, \ldots, y_{\delta}, x\right]\right] /\left(x=y_{1}^{m_{1}}=\cdots=y_{\delta}^{m_{\delta}}\right) \longrightarrow \operatorname{Spf} \mathbb{C}[[x]] .
$$

Here $x$ corresponds to the deformation parameter of the target node, and $y_{i}$ to the deformation parameter of the $i^{\text {th }}$ node of the source curve. (See [Va2, Sect. 2.5] for this deformation-theoretic calculation. This argument can be extended to higher-dimensional targets, and this has been done by many authors; see for example [CH, Va1] in the algebraic category.)

A consequence of J. Li's obstruction theory is that the relationship between $Z_{\text {thick }}$ and $Z_{\text {thin }}$ is "virtually" analogous to (6). More precisely, consider the formal thickening of $Z_{\text {thin }}$ by the formal deformation space of $D_{\Gamma^{\prime}, \Gamma^{\prime \prime}}$ and the formal deformation spaces of the nodes $\left\{N_{i}\right\}$ of the source curve mapping to the singularity of $D_{\Gamma^{\prime}, \Gamma^{\prime \prime}}$. Locally on $Z_{\text {thin }}$, $x$ is a generator of the first deformation space, and $y_{1}, \ldots, y_{\delta}$ are generators of the rest. Then $Z_{\text {thick }}$ is the pullback of $x=y_{1}^{m_{1}}=\cdots=y_{\delta}^{m_{\delta}}=0$, and $Z_{\text {thin }}$ is the pullback of $x=y_{1}=\cdots=y_{\delta}=0$. Li describes these line bundles explicitly, but this description is unnecessary for our argument (even though the torus acts nontrivially on them). Both $Z_{\text {thick }}$ and $Z_{\text {thin }}$ sit in a space of virtual dimension $\delta$ larger, corresponding (again étalelocally) to

$$
\operatorname{Spec} \mathcal{O}_{Z_{\text {thin }}} \hookrightarrow \operatorname{Spec} \mathcal{O}_{Z_{\text {thin }}}\left[y_{1}, \ldots, y_{\delta}, x\right] /\left(x=y_{1}^{m_{1}}=\cdots=y_{\delta}^{m_{\delta}}\right) \hookrightarrow \operatorname{Spec} \mathcal{O}_{Z_{\text {thin }}}\left[\left[y_{1}, \ldots, y_{\delta}, x\right]\right] .
$$

The larger space has a natural obstruction theory. The obstruction theory of $Z_{\text {thin }}$ is obtained by capping with $\delta$ Cartier divisors $y_{1}=\cdots=y_{\delta}=0$, and the obstruction theory 
of $Z_{\text {thick }}$ is obtained by capping with $\delta$ Cartier divisors $y_{1}^{m_{1}}=\cdots=y_{\delta}^{m_{\delta}}=0$. (This requires elaboration, as one cannot do this for a general perfect obstruction theory. For a justification, see [Li2, Sect. 4.4].)

Thus the fixed part of the obstruction theory of $Z_{\text {thin }}$ (restricted to $\overline{\mathcal{F}}_{\Gamma^{\prime}, \Gamma^{\prime \prime}}$ ) agrees with the fixed part of the obstruction theory of the larger space (restricted to $\overline{\mathcal{F}}_{\Gamma^{\prime}, \Gamma^{\prime \prime}}$ ), which agrees with the fixed part of the obstruction theory of $Z_{\text {thick }}$ (restricted to $\left.\overline{\mathcal{F}}_{\Gamma^{\prime}, \Gamma^{\prime \prime}}\right)$.

This analysis (or [Li2, Lemma 3.12]) also implies that $\left[Z_{\text {thin }}\right]^{\text {vir }}=\frac{1}{\prod m_{i}}\left[Z_{\text {thick }}\right]^{\text {vir }}$, so the relative codimension of $Z_{\text {thin }}$ in $Z_{\text {thick }}$ is 0 , and the Euler class of the virtual normal bundle is $1 / \prod m_{i}$.

We remark that $Z_{\text {thick }}$ is thus virtually Cartier on $\overline{\mathcal{M}}_{\Gamma}(X, D)$, locally cut out by one equation, but that $Z_{\text {thin }}$ is not: in some sense it is cut out by $(\delta+1)-\delta$ equations.

The inclusion $\overline{\mathcal{F}}_{\Gamma^{\prime}, \Gamma^{\prime \prime}} \subset Z_{\text {thin }}$. We use the gluing version of the obstruction theory of $Z_{\text {thin }}$. By pullback of obstruction theories, the virtual normal bundle of

$$
\overline{\mathcal{M}}_{\Gamma^{\prime}}(X, D)^{\text {simple }} \times_{D^{n}} \overline{\mathcal{M}}_{\Gamma^{\prime \prime}}\left(Y, D_{0}, D_{\infty}\right) \sim \quad \text { in } \quad \overline{\mathcal{M}}_{\Gamma^{\prime}}(X, D) \times_{D^{n}} \overline{\mathcal{M}}_{\Gamma^{\prime \prime}}\left(Y, D_{0}, D_{\infty}\right) \sim
$$

is the pullback of the virtual normal bundle of $\overline{\mathcal{M}}_{\Gamma^{\prime}}(X, D)^{\text {simple }}$ in $\overline{\mathcal{M}}_{\Gamma^{\prime}}(X, D)$, i.e. $N_{\Gamma^{\prime}}$. By descending by $g l$, we see that the normal bundle of $\overline{\mathcal{F}}_{\Gamma^{\prime}, \Gamma^{\prime \prime}}$ in $Z_{\text {thin }}$ is $N_{\Gamma^{\prime}}$. By definition of the virtual normal bundle of $\overline{\mathcal{M}}_{\Gamma}(X, D)^{\text {simple }}$ in $\overline{\mathcal{M}}_{\Gamma^{\prime}}(X, D)$ (i.e. arising from the non-fixed part of the obstruction theory), the virtual normal bundle has no fixed part.

In conclusion, the obstruction theory of $\overline{\mathcal{F}}_{\Gamma^{\prime}, \Gamma^{\prime \prime}}$ is indeed the fixed part of the restriction of the obstruction theory of $\overline{\mathcal{M}}_{X, D}$, and the Euler class of its virtual normal bundle is $(w-\psi) \times\left(1 / \prod m_{i}\right) \times e\left(N_{\Gamma^{\prime}}\right)$ as desired, completing the proof of relative virtual localization (Theorem 3.6).

\section{VANISHING OF TAUTOLOGICAL CLASSES ON MODULI SPACES OF CURVES AND STRATIFICATION BY NUMBER OF RATIONAL COMPONENTS (THEOREM $\star$ )}

In this section, we give background on the moduli space of curves and its tautological ring. We then define Hurwitz cycles (in the Chow ring of the moduli space of curves), and show that Hurwitz cycles satisfy the conclusion of Theorem $\star$, using degeneration techniques. The proof of Theorem $\star$ will then follow by showing that tautological classes are essentially linear combinations of Hurwitz cycles, using relative virtual localization.

4.1. Background on the moduli space of curves. (See [Va3] for a more leisurely survey of the facts we will need about the moduli space of curves and its tautological ring.) We assume familiarity with $\overline{\mathcal{M}}_{g, n}$.

There are natural morphisms among moduli spaces of stable curves, forgetful morphisms

$$
\overline{\mathcal{M}}_{g, n} \longrightarrow \overline{\mathcal{M}}_{g, n-1}
$$


and gluing morphisms

$$
\begin{aligned}
\overline{\mathcal{M}}_{g_{1}, n_{1}+1} \times \overline{\mathcal{M}}_{g_{2}, n_{2}+1} & \longrightarrow \overline{\mathcal{M}}_{g_{1}+g_{2}, n_{1}+n_{2}}, \\
\overline{\mathcal{M}}_{g, n+2} & \longrightarrow \overline{\mathcal{M}}_{g+1, n} .
\end{aligned}
$$

Gluing morphisms will be denoted by $g l$. The tautological ring may be defined in terms of the natural morphisms as follows.

4.2. Definition. The system of tautological rings are defined as the smallest system of $\mathbb{Q}$-vector spaces $\left(R^{i}\left(\overline{\mathcal{M}}_{g, n}\right)\right)_{i, g, n}$ satisfying:

- $\psi_{1}^{a_{1}} \cdots \psi_{n}^{a_{n}} \subset R^{*}\left(\overline{\mathcal{M}}_{g, n}\right)$, and

- the system is closed under pushforwards by the natural morphisms.

(Throughout we consider Chow groups with $\mathbb{Q}$-coefficients.) The tautological ring of a dense open subset of $\overline{\mathcal{M}}_{g, n}$ is defined by restriction. Let $R_{j}\left(\overline{\mathcal{M}}_{g, n}\right)=R^{\operatorname{dim} \overline{\mathcal{M}}_{g, n}-j}\left(\overline{\mathcal{M}}_{g, n}\right)$ be the group of dimension $j$ tautological classes.

This is equivalent to the other definitions of the tautological ring appearing in the literature. For example, to show equivalence with the definition of [GrPa2], by [GrPa2, Prop. 11] it suffices to show that any monomial in the $\psi$-classes and $\kappa$-classes lies in the groups defined in Definition 4.2. But such a class is clearly the pushforward of a monomial in $\psi$-classes on a space of curves with more points (as in [Lo, p. 413]).

Let $\mathcal{S}_{k}$ (resp. $\mathcal{S}_{\geq k}$, etc.) be the union of strata of $\overline{\mathcal{M}}_{g, n}$ with precisely $k$ (resp. at least $k$, etc.) components of geometric genus 0 . Then $A_{*}\left(\mathcal{S}_{\geq k}\right) \rightarrow A_{*}\left(\overline{\mathcal{M}}_{g, n}\right) \rightarrow A_{*}\left(\mathcal{S}_{<k}\right) \rightarrow 0$ is exact. Let $I_{\geq k}$ be the image of $A_{*}\left(\mathcal{S}_{\geq k}\right)$ in $A_{*}\left(\overline{\mathcal{M}}_{g, n}\right)$; it is an ideal of $A^{*}\left(\overline{\mathcal{M}}_{g, n}\right)$. Then Theorem $\star$ is equivalent to $R^{i}\left(\overline{\mathcal{M}}_{g, n}\right) \subset I_{\geq i-g+1}$. (It will be useful to observe that the image of $I_{\geq k}$ under a forgetful morphism is $I_{\geq k-1}$.)

Define $\widetilde{\mathcal{M}}_{g, n}$ in the same way as $\overline{\mathcal{M}}_{g, n}$, except the curve is not required to be connected. Then $\widetilde{\mathcal{M}}_{g, n}$ is a Deligne-Mumford stack of finite type, and is stratified by $\mathcal{S}_{k}$ analogously; each irreducible component is isomorphic to the quotient of a product of $\overline{\mathcal{M}}_{g^{\prime}, n^{\prime}}$ s by a finite group. Let $\widetilde{\mathcal{M}}=\coprod_{g, n: 2 g-2+n>0} \widetilde{\mathcal{M}}_{g, n}$.

4.3. Remark. Note that Theorem $\star$ is true for given $g, n$ with $\overline{\mathcal{M}}$ replaced by $\widetilde{\mathcal{M}}$ if it is true for all $\overline{\mathcal{M}}_{g^{\prime}, n^{\prime}}$ with $\operatorname{dim} \overline{\mathcal{M}}_{g^{\prime}, n^{\prime}} \leq \operatorname{dim} \overline{\mathcal{M}}_{g, n}$. Hence Theorem $\star$ implies that the corresponding statement with $\overline{\mathcal{M}}$ replaced by $\widetilde{\mathcal{M}}$ is true in general.

4.4. Hurwitz classes, and their behavior with respect to the stratification. For the rest of Section 4, fix $g$ and $n$. Following Ionel, define a trivial cover of $\left(\mathbb{P}^{1}, 0, \infty\right)$ to be a map of the form $\mathbb{P}^{1} \rightarrow \mathbb{P}^{1},[x ; y] \mapsto\left[x^{u} ; y^{u}\right]$, or equivalently a map from an irreducible curve to $\left(\mathbb{P}^{1}, 0, \infty\right)$ with no branch point away from 0 and $\infty$. A trivial component of a stable relative map is a connected component of the source curve, such that the stabilization of the morphism from that component is a trivial cover. 
Let $\overline{\mathcal{M}}_{g, \alpha}\left(\mathbb{P}^{1}\right)$ be the stack parametrizing degree $d$ stable relative maps from a curve of arithmetic genus $g$ to $\mathbb{P}^{1}$, relative to one point $\infty$, corresponding to partition $\alpha \vdash d$. (As stated earlier, curves are not assumed to be connected, and partitions are taken to be ordered, i.e. the parts are labeled.) Let $\overline{\mathcal{M}}_{g, \alpha, \beta}\left(\mathbb{P}^{1}\right)$ be the moduli space of stable relative maps relative to two points 0 and $\infty$ (with corresponding partitions $\alpha \vdash d$ and $\beta \vdash d$ respectively). Let $\overline{\mathcal{M}}_{g, \alpha, \beta}\left(\mathbb{P}^{1}\right)_{\sim}$ be the analogous space of maps to a non-rigid $\mathbb{P}^{1}$ (Section 2.4).

We will make repeated use of the following result.

4.5. Theorem (Degeneration formula, special case of [Li2, Thm. 3.15]). -

$$
\left[\overline{\mathcal{M}}_{g, \alpha, \beta}\left(\mathbb{P}^{1}\right)\right]^{v i r}=\bigoplus \frac{\prod m_{i}}{\operatorname{Aut}(m .)} g l_{*}\left(\left[\overline{\mathcal{M}}_{g^{\prime}, \alpha, m .}\left(\mathbb{P}^{1}\right)\right]^{v i r} \otimes\left[\overline{\mathcal{M}}_{g^{\prime \prime}, m, \beta}\left(\mathbb{P}^{1}\right)\right]^{v i r}\right)
$$

where the sum on the right is over all possible choices of $g^{\prime}, g^{\prime \prime}, m$., and the $\alpha$ and $\beta$ may be omitted.

This equality should be interpreted in the total space of the family of relative stable map spaces associated to a family of $\mathbb{P}^{1}$ s degenerating to a pair of $\mathbb{P}^{1}$ s meeting in a point. In practice, we will use it by capping these virtual classes against natural Chow cohomology classes that make sense for such a family, and pushing the resulting equality forward to a moduli space of curves.

(We are grateful to Y. Ruan for pointing out that in the analytic setting, one can read off the degeneration formula for virtual fundamental classes from the first four lines of the (earlier) proof of Theorem 5.6 in [LR]. He further remarks that the virtual neighborhood is a much stronger notion than the virtual fundamental class because it is a 'smooth object'.)

Let $r=\operatorname{vdim}\left(\overline{\mathcal{M}}_{g, \alpha}\left(\mathbb{P}^{1}\right)\right)=d+n+2 g-2$ (where vdim denotes virtual dimension). Note that $r$ is the dimension of the open set $\mathcal{M}_{g, \alpha}\left(\mathbb{P}^{1}\right)$ parametrizing maps with smooth source (the Hurwitz scheme). There is a branch morphism $b r: \overline{\mathcal{M}}_{g, \alpha}\left(\mathbb{P}^{1}\right) \rightarrow \operatorname{Sym}^{r} \mathbb{P}^{1}$, obtained by considering the morphisms in $\overline{\mathcal{M}}_{g, \alpha}\left(\mathbb{P}^{1}\right)$ as morphisms to $\mathbb{P}^{1}$, and noting that the image of the Fantechi-Pandharipande branch morphism [FnPa, Thm. 1] to Sym ${ }^{r+\sum\left(\alpha_{i}-1\right)} \mathbb{P}^{1}$ always contains $\infty$ with multiplicity at least $\sum\left(\alpha_{i}-1\right)$.

Define a pushforward morphism $\pi$ from $A_{*}\left(\overline{\mathcal{M}}_{g, \alpha, \beta}\left(\mathbb{P}^{1}\right)\right)$ (and $A_{*}\left(\overline{\mathcal{M}}_{g, \alpha, \beta}\left(\mathbb{P}^{1}\right)_{\sim}\right)$ ) to $A_{*}(\widetilde{\mathcal{M}})$ as follows. On the locus of $\overline{\mathcal{M}}_{g, \alpha, \beta}$ where the cover has no trivial parts, $\pi$ is the usual pushforward (by the morphism induced by the universal curve; the marked points correspond to the parts of $\alpha$ and $\beta$ ). On the locus where the cover has trivial parts, ignore the points on the trivial parts and push forward. (Note that the automorphism groups of the trivial covers still play a role, as they contribute a multiplicity to the virtual fundamental class $\left[\overline{\mathcal{M}}_{\alpha, \beta}\left(\mathbb{P}^{1}\right)\right]^{\text {vir }}$ : the inverse of the product of their degrees.) This definition is designed so that the degeneration formula for fundamental classes (Theorem 4.5) agrees with the gluing of strata (used in Lemma 4.8 and Proposition 4.9 below). 
Now assume $r \geq j$. Define the Hurwitz class $\mathbb{H}_{j}^{g, \alpha}$ informally by considering $\left[\overline{\mathcal{M}}_{g, \alpha}\left(\mathbb{P}^{1}\right)\right]^{\mathrm{vir}}$, fixing $r-j$ branch points (leaving a class of dimension $j$ ), and pushing forward to $\widetilde{\mathcal{M}}$. More precisely,

$$
\mathbb{H}_{j}^{g, \alpha}=\pi_{*}\left(\cap_{s=1}^{r-j} b r^{*}\left(L_{p_{s}}\right) \cap\left[\overline{\mathcal{M}}_{g, \alpha}\left(\mathbb{P}^{1}\right)\right]^{\mathrm{vir}}\right),
$$

where $p_{1}, \ldots, p_{r-j} \in \mathbb{P}^{1}$, and $L_{p}$ corresponds to the hyperplane in $\operatorname{Sym}^{r}\left(\mathbb{P}^{1}\right)$ of $r$-tuples containing $p$.

Define the double Hurwitz class $\mathbb{H}^{g, \alpha, \beta}$ by considering $\left[\overline{\mathcal{M}}_{g, \alpha, \beta}\left(\mathbb{P}^{1}\right)\right]^{\text {vir }}$, fixing one branch point, and pushing forward to $\widetilde{\mathcal{M}}$ :

$$
\mathbb{H}^{g, \alpha, \beta}=\pi_{*}\left(b r^{*}\left(L_{1}\right) \cap\left[\overline{\mathcal{M}}_{g, \alpha, \beta}\left(\mathbb{P}^{1}\right)\right]^{\mathrm{vir}}\right) \in A_{*}(\widetilde{\mathcal{M}}) .
$$

Define $\mathbb{H}_{\sim}^{g, \alpha, \beta}$ similarly by

$$
\mathbb{H}_{\sim}^{g, \alpha, \beta}=\pi_{*}\left[\overline{\mathcal{M}}_{g, \alpha, \beta}\left(\mathbb{P}^{1}\right)_{\sim}\right]^{\mathrm{vir}} \in A_{*}(\widetilde{\mathcal{M}}) .
$$

Then $\operatorname{dim} \mathbb{H}^{g, \alpha, \beta}=\operatorname{dim} \mathbb{H}_{\sim}^{g, \alpha, \beta}=r(g, \alpha, \beta)-1$, where $r(g, \alpha, \beta)=\operatorname{vdim}\left(\overline{\mathcal{M}}_{g, \alpha, \beta}\left(\mathbb{P}^{1}\right)\right)$ is the "expected number" of branch points away from 0 and $\infty$.

Note that $\mathbb{H}_{j}^{g, \alpha}$ is the pushforward of a class on $\cap_{s=1}^{r-j} b r^{-1}\left(L_{p_{s}}\right) \cap \overline{\mathcal{M}}_{g, \alpha}\left(\mathbb{P}^{1}\right)$, and similarly for the double Hurwitz class $\mathbb{H}^{g, \alpha, \beta}$.

\subsection{Lemma. $-\mathbb{H}^{g, \alpha, \beta}=r(g, \alpha, \beta) \mathbb{H}_{\sim}^{g, \alpha, \beta}$.}

Proof. (The commutative diagram below may be helpful.) Let $T$ be the smooth locus of the universal target over $\overline{\mathcal{M}}_{g, \alpha, \beta}\left(\mathbb{P}^{1}\right)_{\sim}$, minus the 0 - and $\infty$-sections. Let $B$ the branch locus of the universal map (a subset of $T$ ). Then $B$ is an effective Cartier divisor on $T$, finite of degree $r(g, \alpha, \beta)$ over the base (the arguments of [FnPa, Sect. 3] for stable maps apply without change for stable relative maps). Then $T$ is canonically an open subset of $\overline{\mathcal{M}}_{g, \alpha, \beta}\left(\mathbb{P}^{1}\right)$ (using the three points $0, \infty$, and the point on the universal target to give a morphism to $\mathbb{P}^{1}$, sending the universal point to 1 ), and their obstruction theories are identical. Indeed, this open set of $\overline{\mathcal{M}}_{g, \alpha, \beta}\left(\mathbb{P}^{1}\right)$ is just the corresponding open subset of the base-change of $\overline{\mathcal{M}}_{g, \alpha, \beta}\left(\mathbb{P}^{1}\right)_{\sim}$ by the forgetful morphism from $\mathcal{T}$ to $\mathcal{T}_{\sim}$. Since the virtual classes are constructed from relative perfect obstruction theories over $\mathcal{T}$ and $\mathcal{T}_{\sim}$ respectively, this follows from the base change property of the virtual class construction. Thus, the restriction of $\left[\overline{\mathcal{M}}_{g, \alpha, \beta}\left(\mathbb{P}^{1}\right)\right]^{\text {vir }}$ to $T$ is just the flat pull-back of $\left[\overline{\mathcal{M}}_{g, \alpha, \beta}\left(\mathbb{P}^{1}\right)_{\sim}\right]^{\text {vir. }}$. Under this open immersion, the effective Cartier divisor $B$ on $T$ is canonically the effective Cartier divisor $b r^{-1}\left(L_{1}\right)$. Since the degree of the morphism $B \rightarrow \overline{\mathcal{M}}_{g, \alpha, \beta}\left(\mathbb{P}^{1}\right)_{\sim}$ is $r(g, \alpha, \beta)$, the result follows.

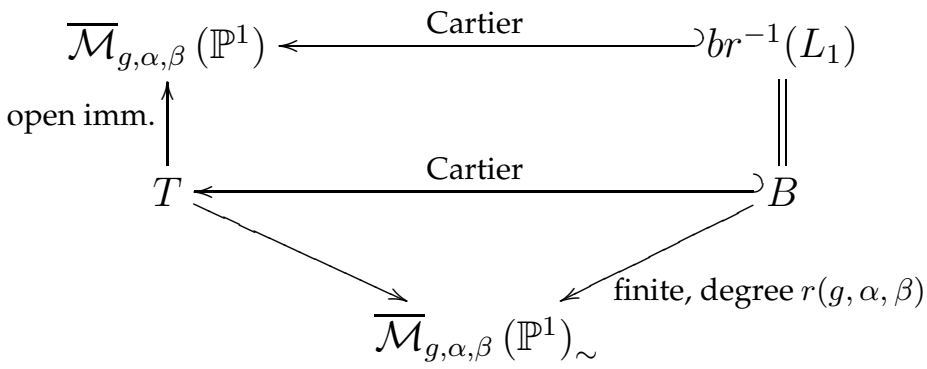




\subsection{Lemma. - If $(g, \alpha, \beta) \neq(0,(d),(d))$, then $\mathbb{H}^{g, \alpha, \beta} \in I_{\geq 1}$.}

We are grateful to E.-N. Ionel for pointing out this result to us. The proof below is extracted from her proof of [I, Prop. 2.8], with the following differences: this argument is in the virtual setting; the source curve is not required to be connected, and the Ionel-Parker gluing formula is replaced by the J. Li degeneration formula (Theorem 4.5). In particular, the key trick in the following argument (to use a surprising forgetful morphism) is due to her.

Proof. We argue by induction on $\operatorname{dim} \mathbb{H}^{g, \alpha, \beta}$, and then on the degree $d=|\alpha|$. Assume that we know the result for $\left(g^{\prime}, \alpha^{\prime}, \beta^{\prime}\right)$ with $\operatorname{dim} \mathbb{H}^{g^{\prime}, \alpha^{\prime}, \beta^{\prime}}<\operatorname{dim} \mathbb{H}^{g, \alpha, \beta}$, and for $\operatorname{dim} \mathbb{H}^{g^{\prime}, \alpha^{\prime}, \beta^{\prime}}=$ $\operatorname{dim} \mathbb{H}^{g, \alpha, \beta}$ but $\left|\alpha^{\prime}\right|<|\alpha|$. Consider $e v^{-1}(p) \cap \overline{\mathcal{M}}_{g, \alpha, \beta, 1}\left(\mathbb{P}^{1}\right)$, parametrizing relative stable maps of the sort we are interested in, with a branch point fixed at 1 , and with an additional marked point $q_{1}^{\prime}$ mapping to some fixed $p \in \mathbb{P}^{1}$. Let $\mathbb{H}$ be its virtual fundamental class. Let $\rho$ be the forgetful morphism forgetting $q_{1}$, the marked point corresponding to $\alpha_{1}$. By breaking the class $\mathbb{H}$ in two ways, we will show that $\rho_{*} \pi_{*} \mathbb{H} \in I_{\geq 1}$, and that $\rho_{*} \pi_{*} \mathbb{H}$ is a non-zero multiple of $\mathbb{H}^{g, \alpha, \beta}$ modulo $I_{\geq 1}$, from which the result follows.

Degenerate $\mathbb{H}$ by breaking the target into two pieces, so that 0 and 1 lie on $T_{1}$, say, and $p$ and $\infty$ lie on $T_{2}$. By the degeneration formula (Theorem 4.5), we can express $\mathbb{H}$ as the sum of other double Hurwitz classes, appropriately glued. Consider one such summand $g l\left(\mathbb{H}_{1} \otimes \mathbb{H}_{2}\right)$, where $\mathbb{H}_{i}$ corresponds to the stable relative map to $T_{i}$. The cover of $T_{1}$ has branching away from the two special points 0 and $T_{1} \cap T_{2}$, as it has branching above 1 . If the cover of $T_{2}$ also has branching away from its two special points $T_{1} \cap T_{2}$ and $\infty$, then by the inductive hypothesis $\pi_{*}\left(\mathbb{H}_{1}\right) \in I_{\geq 1}$, and also $\pi_{*}\left(\mathbb{H}_{2}\right) \in I_{\geq 1}$ (by applying the inductive hypothesis to the map without the marked point $q_{1}^{\prime}$, and then pulling back by the forgetful morphism; we use here the pullback property of the virtual fundamental class). Thus $\pi_{*}(\mathbb{H}) \in I_{\geq 2}$, from which $\rho_{*} \pi_{*}(\mathbb{H}) \in I_{\geq 1}$. On the other hand, if the cover of $T_{2}$ has no branching away from the two special points, then we can immediately identify a three-pointed rational curve in the stabilization of the source: the component mapping to $T_{2}$ containing $q_{1}^{\prime}$, one of the marked points mapping to $\infty$, and a node mapping to $T_{1} \cap T_{2}$. The one exception is if this node over $T_{1} \cap T_{2}$ is glued to a trivial cover of $T_{1}$, with the point above 0 marked $q_{1}$, as then once $q_{1}$ is forgotten, this component is stabilized away. But in this case, the inductive hypothesis for covers of lower degree implies that the contribution from the remainder of the curve mapping to $T_{1}$ lies in $I_{\geq 1}$. Hence we have shown that $\rho_{*} \pi_{*} \mathbb{H} \in I_{\geq 1}$.

Next, degenerate $\mathbb{H}$ by breaking the target so that 0 and $p$ lie in $T_{1}$ and 1 and $\infty$ lie in $T_{2}$. We proceed as in the previous paragraph. Consider any summand $g l\left(\mathbb{H}_{1} \otimes \mathbb{H}_{2}\right)$ in the degeneration formula. If there is branching over $T_{1}$ away from the two special points, then this summand lies in $I_{\geq 1}$ by the same argument. If there is no other branching over $T_{1}$, and $q_{1}^{\prime}$ lies in a component of the source containing another marked point $q_{i}$ above 0 where $i \neq 1$, then as before we can identify a rational curve in the stabilization of the source, so this summand lies in $I_{\geq 1}$ again. The only remaining case is if there is no branching over $T_{1}$, and $q_{1}^{\prime}$ lies in the component of the source containing $q_{1} ;$ in this case, the contribution 


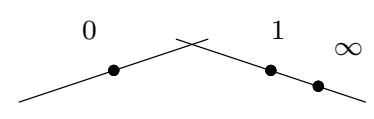

FIGURE 2. $\psi_{0}$ on $\mathcal{T} \subset \mathfrak{M}_{0,3}$

is precisely $\mathbb{H}^{g, \alpha, \beta}$ times the degree of the trivial cover of $T_{1}$ containing $q_{1}^{\prime}$ and $q_{1}$. Hence $\rho_{*} \pi_{*} \mathbb{H}$ is a non-zero multiple of $\mathbb{H}^{g, \alpha, \beta}$ modulo $I_{\geq 1}$, from which the result follows.

4.8. Lemma. - $\psi^{k} \cap \mathbb{H}_{\sim}^{g, \alpha, \beta} \in I_{\geq k+1}$.

Here $\psi$ is the class defined in Section 2.5, and $\psi^{k} \cap \mathbb{H}_{\sim}^{g, \alpha, \beta}$ should be interpreted as $\pi_{*}\left(\psi^{k} \cap\left[\overline{\mathcal{M}}_{g, \alpha, \beta}\left(\mathbb{P}^{1}\right)_{\sim}\right]^{\text {vir }}\right)$.

Proof. We prove the result by induction. The case $k=0$ follows from the previous two lemmas. Assume now that $k>0$. By Lemma 4.6 it suffices to show the analogous result on $b r^{-1}\left(L_{1}\right) \cap \overline{\mathcal{M}}_{g, \alpha, \beta}\left(\mathbb{P}^{1}\right)$. Via the target (with three marked sections $0,1, \infty$ ), this space admits a morphism to $\overline{\mathfrak{M}}_{0,3}$, and $\psi$ is pulled back from $\psi_{0}$ on $\mathcal{T} \subset \overline{\mathfrak{M}}_{0,3}$. Now $\psi_{0}$ is equivalent to a boundary divisor. (This is not true on $\overline{\mathfrak{M}}_{0,2}$, hence the necessity of lifting to $\overline{\mathcal{M}}_{g, \alpha, \beta}\left(\mathbb{P}^{1}\right)$ instead of working on $\overline{\mathcal{M}}_{g, \alpha, \beta}\left(\mathbb{P}^{1}\right)_{\sim}$ !) More precisely, $\psi_{0}$ is the boundary divisor corresponding to (degenerations of) the curves shown in Figure 2, so

$$
\psi^{k} \cap \mathbb{H}_{\sim}^{g, \alpha, \beta}=\sum g l\left(\left(\psi^{k-1} \cap \mathbb{H}_{\sim}^{g^{\prime}, \alpha^{\prime}, \beta^{\prime}}\right) \otimes \mathbb{H}^{g^{\prime \prime}, \alpha^{\prime \prime}, \beta^{\prime \prime}}\right) .
$$

But $\psi^{k-1} \cap \mathbb{H}_{\sim}^{g^{\prime}, \alpha^{\prime}, \beta^{\prime}} \in I_{\geq k}$ by the inductive hypothesis, and $\mathbb{H}^{g^{\prime \prime}, \alpha^{\prime \prime}, \beta^{\prime \prime}} \in I_{\geq 1}$ by Lemma 4.7 , so we are done, as the degeneration formula (Theorem 4.5) is compatible under $\pi$ with gluing.

We note that this proof can be unwound to show that $\psi^{k} \cap \mathbb{H}_{\sim}^{g, \alpha, \beta}$ is equivalent to a sum of classes corresponding to covers of a chain of $\mathbb{P}^{1}$ 's.

\subsection{Proposition. $-\mathbb{H}_{j}^{g, \alpha} \in I_{\geq 2 g-2+n-j}$.}

Proof. Choose the points $\left\{p_{s}\right\}$ to be distinct. The class $\mathbf{H}=b r^{*}\left(\cap_{s=1}^{r-j} L_{p_{s}}\right) \cap\left[\overline{\mathcal{M}}_{g, \alpha}\left(\mathbb{P}^{1}\right)\right]^{\text {vir }}$ is supported on $b r^{-1}\left(\cap L_{p_{s}}\right) \cap\left[\overline{\mathcal{M}}_{g, \alpha}\left(\mathbb{P}^{1}\right)\right]$. Degenerate the target into a chain of $r-j$ components $T_{1}, \ldots, T_{r-j}$, with $p_{s} \in T_{s}$, and $\infty \in T_{r-j}$ (see Figure 3). By the degeneration formula (Theorem 4.5), capped with $b r^{*}\left(L_{p_{s}}\right)$, supported on $b r^{-1}\left(L_{p_{s}}\right)$ ), and the compatibility of the degeneration formula with $\pi, \mathbb{H}_{j}^{g, \alpha}=\pi_{*} \mathbf{H}$ equals a sum with terms of the form

$$
\pi_{*}\left(g l\left(\bigotimes_{s=1}^{r-j} b r^{*}\left(L_{p}\right)\left[\mathcal{M}_{s}\right]^{\mathrm{vir}}\right)\right)=g l\left(\bigotimes_{s=1}^{r-j} \pi_{*}\left(b r^{*}\left(L_{p}\right)\left[\mathcal{M}_{s}\right]^{\mathrm{vir}}\right)\right),
$$

where $\mathcal{M}_{s}$ is the appropriate moduli space of stable relative maps to $T_{s}$. The product is taken over all appropriate matching of points. Note that while $\mathcal{M}_{1}$ is the space of maps to $\mathbb{P}^{1}$ relative to one point, for all $s>1, \mathcal{M}_{s}$ is a space of maps to $\mathbb{P}^{1}$ relative to two points. 

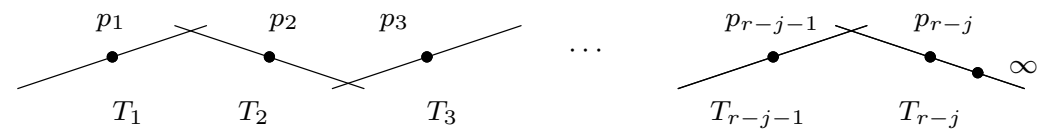

FIGURE 3. Breaking the Hurwitz class by breaking the target

Hence it suffices to show that any one of the terms of form (10) lies in $I_{\geq 2 g-2+n-j}$. Observe that the number of genus 0 components on the stabilization of a prestable curve is at least the number of genus 0 components with at least 3 special points, minus the number of genus 0 components with 1 special point. Then by Lemma 4.7, the contribution of any one of these terms lies in $I_{\geq Q}$, where $Q$ is $r-j-1$ (one for each $\mathbb{H}^{g^{s}, \alpha^{s}, \beta^{s}, 2 \leq s \leq r-j \text { ) }}$ minus at most $d-1$ (for each genus 0 curve in the preimage of $T_{1}$ with one special point; there are at most $d-1$ such components of the preimage of $T_{1}$ ). As $r=d+n+2 g-2$ (by the Riemann-Hurwitz formula), the result follows.

4.10. Proof of Theorem $\star$. The proof of Theorem $\star$ is now a straightforward generalization of that of [GrVa2].

4.11. Reduction to the case of monomials in $\psi$-classes. The statement of Theorem $\star$ behaves well with respect to the natural morphisms: Pushing forward by the forgetful morphism (7) decreases the codimension by 1 , and decreases the number of genus 0 components by at most 1 . Pushing forward the class $a \otimes b$ by the gluing morphism (8) involves adding both the genera and the numbers of genus 0 components; the codimension is the sum of the old codimensions plus 1 . Pushing forward by the gluing morphism (9) preserves number of genus 0 components, and increases both the genus and codimension by 1 . Hence it suffices to prove Theorem $\star$ for monomials in the $\psi$-classes, i.e. that any dimension $j$ monomial in $\psi$-classes lies in $I_{\geq 2 g-2+n-j}$.

4.12. Localization. We proceed by induction on $\operatorname{dim} \overline{\mathcal{M}}_{g, n}$. Apply relative virtual localization (Corollary 3.7) to the component of $\overline{\mathcal{M}}_{g, \alpha}\left(\mathbb{P}^{1}\right)$ where the source curve is required to be connected. (We could just as well work on the whole space, but this restriction will simplify our exposition by avoiding the nonexistent moduli spaces $\overline{\mathcal{M}}_{0,2}$ and $\overline{\mathcal{M}}_{0,1}$.) We cap with $L_{p_{1}}, \ldots, L_{p_{r-j}}$ to calculate $\mathbb{H}_{j, \mathrm{conn}}^{g, \alpha}$ defined to be the restriction of $\mathbb{H}_{j}^{g, \alpha}$ to the component $\overline{\mathcal{M}}_{g, n}$ of $\widetilde{\mathcal{M}}$. Choose weights on $L_{p_{1}}, \ldots, L_{p_{r-j}}$ corresponding to requiring the fixed branch points points $p_{1}, \ldots, p_{r-j}$ to map to 0 . One fixed locus corresponds to all remaining branch points also mapping to 0 : the simple fixed locus. This locus corresponds to an $n$-pointed genus $g$ curve mapping to 0 , glued to $n$ trivial covers of $\mathbb{P}^{1}$. By relative virtual localization, the contribution of this locus is

$$
r !\left(\prod_{i=1}^{n} \frac{\alpha_{i}^{\alpha_{i}}}{\alpha_{i} !}\right)\left[\frac{1-\lambda_{1}+\cdots \pm \lambda_{g}}{\prod_{i}\left(1-\alpha_{i} \psi_{i}\right)}\right]_{j}=r !\left(\prod_{i=1}^{n} \frac{\alpha_{i}^{\alpha_{i}}}{\alpha_{i} !}\right) P_{j}^{g}\left(\alpha_{1}, \ldots, \alpha_{n}\right) .
$$




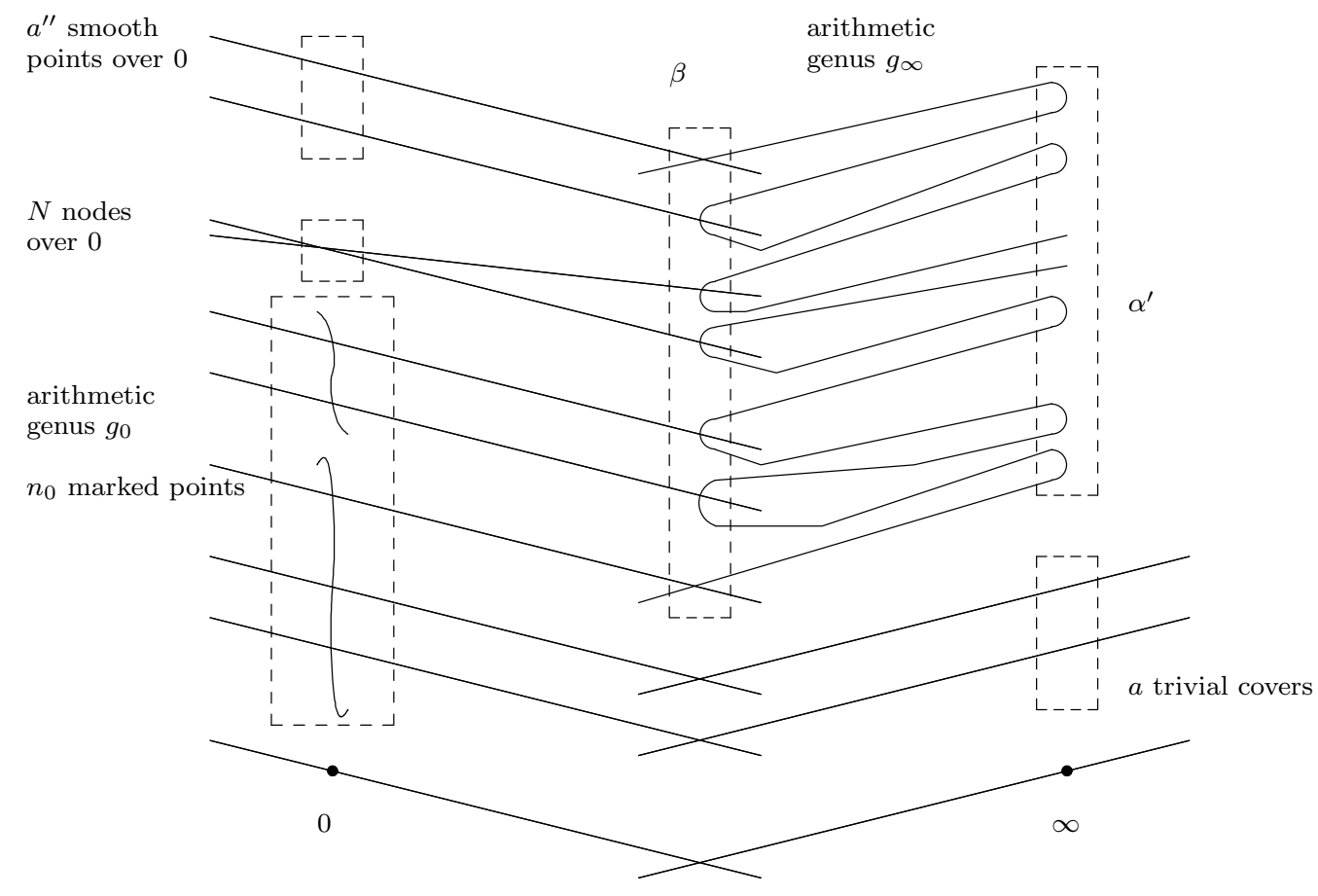

FIGURE 4. An example of a composite fixed locus

( $[x]_{j}$ is the dimension $j$ component of $x$ ) where $P_{j}^{g}$ is a (Chow-valued) polynomial in $n$ variables whose coefficients include all dimension $j$ monomials in $\psi$-classes.

We conclude by showing that the contributions from the remaining fixed loci lie in $I_{\geq 2 g-2+n-j}$. Then as $\mathbb{H}_{j}^{g, \alpha} \in I_{\geq 2 g-2+n-j}$ as well, $P_{j}^{g}\left(\alpha_{1}, \ldots, \alpha_{n}\right) \in I_{\geq 2 g-2+n-j}$. As the coefficients of a polynomial lie in the $\mathbb{Q}$-span of the value of the polynomial evaluated at sufficiently many lattice points, Theorem $\star$ follows.

Consider another (necessarily composite) fixed locus, such as the one depicted in Figure 4. Suppose that the preimage of 0 contains (i) a curve (not necessarily connected) of genus $g_{0}$ with $n_{0}$ marked points, (ii) $N$ nodes, and (iii) $a^{\prime \prime}$ isolated smooth points. Over the non-rigid part of the target ("over $\infty^{\prime \prime}$ ), suppose that there are $a$ trivial covers, and the remaining part of the cover corresponds to a connected component of $\overline{\mathcal{M}}_{g_{\infty}, \beta, \alpha^{\prime}}\left(\mathbb{P}^{1}\right)_{\sim}$, where $\alpha^{\prime}$ is the partition over $\infty$, and $\beta$ is the partition corresponding to the node of the target.

By relative virtual localization, the contribution of this fixed locus is a linear combination of terms of the form $g l\left(\mathbf{c}_{0} \otimes \mathbf{c}_{\infty}\right)$ where

$$
\mathbf{c}_{\infty}=\pi_{*}\left(\psi^{k} \cap\left[\overline{\mathcal{M}}_{g_{\infty}, \beta, \alpha^{\prime}}\left(\mathbb{P}^{1}\right)_{\sim}\right]^{\mathrm{vir}}\right) \in A_{\mathrm{vdim}-k}\left(\overline{\mathcal{M}}_{g_{\infty},|\beta|+\left|\alpha^{\prime}\right|}\right)
$$

and

$$
\mathbf{c}_{0} \in R_{j-(\operatorname{vdim}-k)}\left(\widetilde{\mathcal{M}}_{g_{0}, n_{0}}\right)
$$

where $\operatorname{vdim}=\operatorname{vdim}\left(\overline{\mathcal{M}}_{g_{\infty}, \beta, \alpha^{\prime}}\left(\mathbb{P}^{1}\right)_{\sim}\right)=|\beta|+\left|\alpha^{\prime}\right|+2 g_{\infty}-3$. 
By Lemma 4.8, $\mathbf{c}_{\infty} \in I_{\geq k+1}$, and by the inductive hypothesis and Remark 4.3, $\mathbf{c}_{0} \in$ $I_{\geq 2 g_{0}-2+n_{0}-(j-(\operatorname{vdim}-k))}$. Thus we wish to show that

$$
(k+1)+\left(2 g_{0}-2+n_{0}-j+|\beta|+\left|\alpha^{\prime}\right|+2 g_{\infty}-3-k\right) \geq 2 g-2+n-j .
$$

By comparing the genus of the source curve to that of its components, we have

$$
\left(g_{0}-1\right)+\left(g_{\infty}-1\right)-N-a^{\prime \prime}+|\beta|=g-1 .
$$

By counting preimages of the node of the target in two ways,

$$
n_{0}+2 N+a^{\prime \prime}=|\beta|+a .
$$

Finally, the number of preimages of $\infty$ is

$$
a+\left|\alpha^{\prime}\right|=n .
$$

By adding twice the first equation to the other two, we have

$$
\left((k+1)+\left(2 g_{0}-2+n_{0}-j+|\beta|+\left|\alpha^{\prime}\right|+2 g_{\infty}-3-k\right)\right)-a^{\prime \prime}=2 g-2+n-j
$$

from which (11) follows. Thus $g l\left(\mathbf{c}_{0} \otimes \mathbf{c}_{\infty}\right) \in I_{\geq 2 g+|\alpha|-2-j}$ as desired.

\section{APPLicAtions OF THEOREM *}

We give applications of Theorem $\star$ to prove and extend various theorems and conjectures. We will use the bijection between strata of $\overline{\mathcal{M}}_{g, n}$ and stable graphs, and the "crossratio" or WDVV relation among strata. We also will make repeated use of the following.

5.1. Corollary. - Any $c \in R_{j}\left(\overline{\mathcal{M}}_{g, n}\right)$ is the pushforward (under inclusion) of classes supported on boundary strata corresponding to curves with components of geometric genus $g_{k}$ and $n_{k}$ special points (marked points and node-branches), where

$$
\sum_{k}\left(g_{k}-1+\delta_{g_{k}, 0}\right)+\sum_{k}\left(2 g_{k}-2+n_{k}-\delta_{g_{k}, 0}\right) \geq j \geq \sum_{k}\left(2 g_{k}-2+n_{k}-\delta_{g_{k}, 0}\right) .
$$

(As usual, $\delta_{g_{k}, 0}=1$ if $g_{k}=0$, and 0 otherwise.) Note that $2 g_{k}-2+n_{k}-\delta_{g_{k}, 0} \geq 0$ with equality if and only if $g_{k}=0$ and $n_{k}=3$. Also, if $g_{k}=0$ or 1 for all $k$, then the contribution of this stratum to $c$ is a multiple of the fundamental class of the stratum.

Proof. The left inequality is the dimension of the stratum. By Theorem $\star$,

$$
\sum \delta_{g_{k}, 0} \geq(3 g-3+n-j)-g+1=2 g-2+n-j=\sum\left(2 g_{k}-2+n_{k}\right)-j,
$$

from which the right inequality follows.

5.2. Getzler's conjecture (Ionel's theorem). In [Ge2, Footnote 1], Getzler conjectured that all degree $g$ monomials in $\psi_{1}, \ldots, \psi_{n}$ vanish on $\mathcal{M}_{g, n}$ if $g>0$. Getzler's conjecture was known in genus 1 (classically), and genus 2 (by work of Mumford and Getzler, see equs. (4) and (5) of [Ge2]). Ionel proved Getzler's conjecture in cohomology [I]. Her argument, rewritten in the language of algebraic geometry, should also prove the conjecture in Chow. 
Theorem $\star$ immediately implies Getzler's conjecture, as well as more: all tautological classes of codimension at least $g$ vanish on $\mathcal{M}_{g, n}$ if $g>0$, and in fact they vanish on the larger open set corresponding corresponding to curves with no rational component.

5.3. Poincaré duality speculations. The next few applications concern three parallel conjectures or speculations. Let $\mathcal{M}_{g, n}^{r t}$ be the moduli space of "curves with rational tails", curves with a smooth component of genus $g$ (i.e. with dual graph with a vertex of genus $g$ ). Let $\mathcal{M}_{g, n}^{c t}$ be the moduli space of "curves of compact type", curves with compact Jacobian (i.e. with dual graph with no loops). Hence

$$
\mathcal{M}_{g, n}^{r t} \subset \mathcal{M}_{g, n}^{c t} \subset \overline{\mathcal{M}}_{g, n} .
$$

5.4. Conjecture (Faber, Looijenga, Pandharipande, et al. [Pa, Sect. 2]). - The space $\overline{\mathcal{M}}_{g, n}$ (resp. $\left.\mathcal{M}_{g, n}^{c t}, \mathcal{M}_{g, n}^{r t}\right)$ "behaves like" a complex variety of dimension $D=3 g-3+n$ (resp. $2 g-3+n$, $g-2+n)$. More precisely,

- Socle statement: $R^{i}=0$ for $i>D, R^{D} \cong \mathbb{Q}$, and

- Perfect pairing statement: for $0 \leq i \leq D$, the natural map $R^{i} \times R^{D-i} \rightarrow R^{D}$ is a perfect pairing.

Although it is not clear if one should expect this strong statement to be true, this speculation has motivated much interesting work.

The case $\mathcal{M}_{g, 0}=\mathcal{M}_{g, 0}^{r t}$ is part of Faber's conjecture [Fb3]. The case $\overline{\mathcal{M}}_{g, n}$ was asked by Hain and Looijenga [HaLo, Question 5.5, p. 108]. The cases when $n=0$ were stated in [FbPa1]. The general statement is likely due to Faber and Pandharipande.

Some evidence for the perfect pairing portion will be given in a later paper [GrVa3]. We now present proofs of the socle statements in all three cases.

5.5. Socle proof for $\overline{\mathcal{M}}_{g, n}$. This argument is essentially the one given in [GrVa2], which can be interpreted as an early version of Theorem $\star$. If $i>3 g-3+n=\operatorname{dim} \overline{\mathcal{M}}_{g, n}, R^{i}\left(\overline{\mathcal{M}}_{g, n}\right)=0$ for dimensional reasons. If $i=3 g-3+n$ then by Corollary 5.1, $R^{i}\left(\overline{\mathcal{M}}_{g, n}\right)$ is generated by the 0 -dimensional strata (whose graphs have $2 g-2+n$ genus 0 trivalent vertices). These strata are rationally equivalent (by judicious use of the cross-ratio relation, or by observing that they lie on the image of the rational space $\overline{\mathcal{M}}_{0,2 g+n}$ under $g$ gluing morphisms (9)). They are non-zero because they have nonzero degree.

5.6. Socle proof for $\mathcal{M}_{g, n}^{c t}$. This is the first genus-free evidence for the "compact type" conjecture. As a bonus, this approach produces a natural generator of the socle.

The argument parallels the previous one. The case $(g, n)=(2,0)$ is immediate. For $(g, n) \neq(2,0)$, any $n$-pointed genus $g$ stable graph with no loops has no more than $g-2+n$ genus 0 vertices, and if equality holds, then all genus 0 vertices are trivalent, and the other vertices are genus 1 "leaves" (see Figure [5] for an example), so in particular Corollary 5.1 


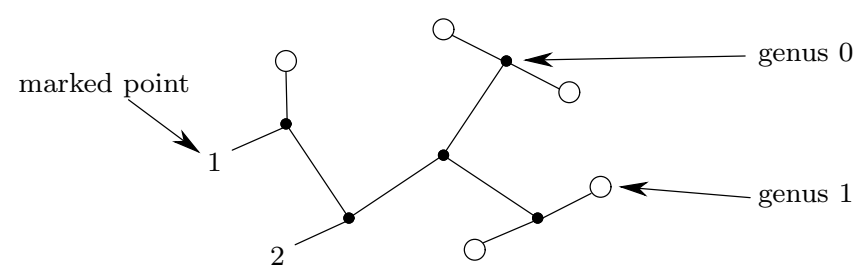

FIGURE 5. An example of a generator of $R^{2 g-3+n}\left(\mathcal{M}_{5,2}^{c t}\right)$

applies. Any such strata is codimension $2 g-3+n$ (dimension $g$ ). Any two such strata are rationally equivalent by the cross-ratio relation, or by using the rationality of the space $\overline{\mathcal{M}}_{0, g+n}$.

Hence by Theorem $\star$, if $i>D=2 g-3+n$, then any element of $R^{i}\left(\mathcal{M}_{g, n}^{c t}\right)$ vanishes on $\mathcal{M}_{g, n}^{c t}$, and any element of $R^{D}\left(\mathcal{M}_{g, n}^{c t}\right)$ is a multiple of (any) one of these strata. This strata is nonzero on $\mathcal{M}_{g, n}^{c t}$ as the integral of $\lambda_{g}$ over its closure is clearly $\left(\int_{\overline{\mathcal{M}}_{1,1}} \lambda_{1}\right)^{g}=1 / 24^{g} \neq 0$, and $\lambda_{g}$ vanishes on $\overline{\mathcal{M}}_{g, n}-\mathcal{M}_{g, n}^{c t}[$ FbPa1, Sect. 0.4].

We remark that these curves are related to flag curves [HMo, p. 246].

5.7. Socle proof for $\mathcal{M}_{g, n}^{r t}$. Conjecture 5.4 is known for $g=0\left([\overline{K e}]\right.$, as $\left.\overline{\mathcal{M}}_{g, 0}=\mathcal{M}_{g, 0}^{r t}\right)$, so we assume $g>0$.

5.8. Proposition. -

(a) For $i>g-2+n, R^{i}\left(\mathcal{M}_{g, n}^{r t}\right)=0$.

(b) For $n>0$, the forgetful morphism $\alpha: R^{g-2+n}\left(\mathcal{M}_{g, n}^{r t}\right) \rightarrow R^{g-1}\left(\mathcal{M}_{g, 1}^{r t}=\mathcal{M}_{g, 1}\right)$ is an isomorphism.

Part (a) for $n=0$ is Looijenga's theorem [Lo]. The Proposition should be deducible from the main theorem of [Lo], although we have not checked the details.

Proof. (a) If $n>0$, then $\mathcal{M}_{g, n}^{r t} \subset \mathcal{S}_{\leq n-1}$, so by Theorem $\star$ any class in $R^{i}\left(\mathcal{M}_{g, n}^{r t}\right)$ is the pushforward of a class supported on $\mathcal{S}_{\geq n} \cap \mathcal{M}_{g, n}^{r t}=\emptyset$. By Definition 4.2 of the tautological ring, $R^{a+1}\left(\mathcal{M}_{g, 1}\right) \rightarrow R^{a}\left(\mathcal{M}_{g}=\mathcal{M}_{g}^{r t}\right)$ is surjective, so the case $n=0$ follows.

(b) Any $[C] \in \overline{\mathcal{M}}_{0, n+1}$ induces a closed immersion $\iota_{C}: \mathcal{M}_{g, 1} \rightarrow \mathcal{M}_{g, n}^{r t}$ via the gluing morphism (8). As $\overline{\mathcal{M}}_{0, n+1}$ is rational, the induced map $A_{*}\left(\mathcal{M}_{g, 1}\right) \rightarrow A_{*}\left(\mathcal{M}_{g, n}^{r t}\right)$ is independent of $C$; denote it by $\beta$. Let $C_{1}, \ldots, C_{s}$ be the curves corresponding to the 0 -dimensional strata of $\overline{\mathcal{M}}_{0, n+1}$. By Theorem $\star$, any $a \in R^{g-2+n}\left(\mathcal{M}_{g, n}^{r t}\right)$ is the pushforward of a class supported on $\mathcal{S}_{n-1} \cap \mathcal{M}_{g, n}^{r t}=\cup_{j} \iota_{C_{j}}\left(\mathcal{M}_{g, 1}\right)$, and hence the pushforward of a class $b$ supported on $\iota_{C_{1}}\left(\mathcal{M}_{g, 1}\right) \cong \mathcal{M}_{g, 1}$. Necessarily $b=\alpha(a)$ (and thus is tautological). Hence $\beta \circ \alpha$ is the identity on $R^{*}\left(\overline{\mathcal{M}}_{g, n}\right)$. As $\alpha \circ \beta$ is clearly the identity on $R^{*}\left(\mathcal{M}_{g, 1}\right)$, we are done. 
In light of Faber's calculation $\operatorname{deg} \kappa_{g-2} \lambda_{g} \lambda_{g-1} \neq 0$ and that $\lambda_{g} \lambda_{g-1}$ vanishes on $\overline{\mathcal{M}}_{g}-\mathcal{M}_{g}$ [Fb3, Theorem 2], it now follows that $R^{g-2+n}\left(\mathcal{M}_{g, n}^{r t}\right) \neq 0$ for all $g, n$. Finally, $R^{g-2+n}\left(\mathcal{M}_{g, n}^{r t}\right) \cong$ $\mathbb{Q}$ follows from $R^{g-1}\left(\mathcal{M}_{g, 1}\right)=\left\langle\psi_{1}^{g-1}\right\rangle$, which is known by Looijenga. We do not see how to obtain this result easily using our approach.

5.9. Generalizations of Diaz' theorem. Following [Lo, p. 412], Diaz' theorem [D, p. 79] (there is no complete subvariety of $\mathcal{M}_{g}$ of dimension greater than $g-2$ ) can be extended as follows.

\subsection{Proposition. -}

(a) There is no complete subvariety of $S_{\leq_{s}}$ of dimension greater than $s+g-1$. In particular, there is no complete subvariety of $S_{0}$ of dimension greater than $g-1$.

(b) There is no complete subvariety of $M_{g, n}^{r t}$ of dimension greater than $g-2+n$.

(c) There is no complete subvariety of $M_{g, n}^{c t}$ of dimension greater than $2 g-3+n$.

Here roman letters denote the coarse moduli space. Part (b) is a trivial generalization of Diaz' theorem, and part (c) follows from another result of Diaz [D, Cor. p. 80] ([FbLo] notes that $2 g-1$ should be $2 g-2$ here); we include the argument only as an illustration of how these results are also immediately consequences of Theorem $\star$. Note that the bound in (a) for $s=0$ is necessarily worse than the bound of Diaz' theorem, as there is a complete curve in $S_{0}\left(\overline{\mathcal{M}}_{2,0}\right)$ (e.g. $y^{2}=x(x-1)(x-2)(x-3)(x-4)(x-t)$ ).

Proof. Suppose $V$ is a complete substack of $\mathcal{S}_{\leq s}$ of dimension $d \geq g-1$. Let $\alpha$ be any ample tautological divisor on the coarse moduli space. (The coarse moduli space is projective by Knudsen, and all divisors are tautological by [ACo]. Alternatively, Cornalba gives a tautological ample class explicitly [Co, p. 13].) If $d \geq e \geq g-1$, the class $\alpha^{e}$ is tautological, and by Theorem $\star$ is rationally equivalent to a class supported on $\mathcal{S}_{\geq e-g+1}$. Hence $\mathcal{S}_{\geq e-g+1} \cap V$ contains a nonempty subset of dimension at least $d-e$. The case $d=e=s+g$ yields (a). Parts (b) and Part (c) follow from $M_{g, n}^{r t} \subset S_{\leq n-1}(n \geq 1)$ and $M_{g, n}^{c t} \subset S_{\leq g-2+n}$, or directly from Sections 5.7 and 5.6 respectively.

5.11. Universal description of the tautological groups in low dimension. The tautological groups are completely understood in codimension 1 and 2 ([ACo] and [Po] respectively), but even codimension 3 is combinatorially complicated. Theorem $\star$ implies that the tautological groups are actually more straightforward in low dimension, by providing a parsimonious systems of generators. This is by exploiting the right-hand inequality of Corollary 5.1 for small values of $j$, there are very few possible values of $g_{k}$ and $n_{k}$ that can occur in a boundary stratum. For notational convenience, we will imprecisely denote such a stratum (with components of genus $g_{k}$, with $n_{k}$ special points) by $\prod_{k} \overline{\mathcal{M}}_{g_{k}, n_{k}}$. We will list the consequences of this inequality up through dimension 6 .

Dimension 0. As observed in Section [5.5, $R_{0}\left(\overline{\mathcal{M}}_{g, n}\right)$ is generated by boundary strata of the form $\prod \overline{\mathcal{M}}_{0,3}$; they are all rationally equivalent. 
Dimension 1. $R_{1}\left(\overline{\mathcal{M}}_{g, n}\right)$ is again generated by boundary strata (in which there is one $\overline{\mathcal{M}}_{0,4}$ or $\overline{\mathcal{M}}_{1,1}$ factor). To see this, note that by the right hand inequality of Corollary 5.1 with $j=1$, the only pairs $\left(g_{k}, n_{k}\right)$ that can occur are $(0,3),(0,4)$, or $(1,1)$, and for all but one value of $k$, we must have $\left(g_{k}, n_{k}\right)=(0,3)$. Since these boundary strata have dimension 1 , the only one-dimensional classes they can support are their fundamental classes.

Dimension 2. In dimension 2, Corollary 5.1 allows for the possibility of a non-boundary class: a divisor on $\overline{\mathcal{M}}_{2,0}$. However, by [M, Part III], $A^{1}\left(\overline{\mathcal{M}}_{2,0}\right)$ is generated by boundary strata, so we can conclude that $R_{2}\left(\overline{\mathcal{M}}_{g, n}\right)$ is generated by boundary strata for all $g$ and $n$.

Dimension 3. $R_{3}\left(\overline{\mathcal{M}}_{g, n}\right)$ is generated by boundary strata, together with classes supported on strata corresponding to a product of $\overline{\mathcal{M}}_{2,1}$ with copies of $\overline{\mathcal{M}}_{0,3}$; there is a single nonboundary generator corresponding to $\psi_{1}$ on $\overline{\mathcal{M}}_{2,1}$, as this is the only nonboundary class in $A^{1}\left(\overline{\mathcal{M}}_{2,1}\right)$.

Dimension 4. $R_{4}\left(\overline{\mathcal{M}}_{3,0}\right)$ was computed in [Fb1]. For other $g, n, R_{4}\left(\overline{\mathcal{M}}_{g, n}\right)$ is generated by boundary classes, as well as classes of the form $\psi_{1}$ on either

$$
\overline{\mathcal{M}}_{2,2} \times \prod \overline{\mathcal{M}}_{0,3}, \quad \overline{\mathcal{M}}_{2,1} \times \overline{\mathcal{M}}_{0,4} \times \prod \overline{\mathcal{M}}_{0,3}, \quad \text { or } \quad \overline{\mathcal{M}}_{2,1} \times \overline{\mathcal{M}}_{1,1} \times \prod \overline{\mathcal{M}}_{0,3}
$$

with the $\psi$ class on the genus 2 factor. The last two cases require the facts that $A^{1}\left(\overline{\mathcal{M}}_{2,1} \times \overline{\mathcal{M}}_{0,4}\right) \cong$ $A^{1}\left(\overline{\mathcal{M}}_{2,1}\right) \oplus A^{1}\left(\overline{\mathcal{M}}_{0,4}\right)$ and $A^{1}\left(\overline{\mathcal{M}}_{2,1} \times \overline{\mathcal{M}}_{1,1}\right) \cong A^{1}\left(\overline{\mathcal{M}}_{2,1}\right) \oplus A^{1}\left(\overline{\mathcal{M}}_{1,1}\right)$, which follow from $\overline{\mathcal{M}}_{0,4} \cong \bar{M}_{1,1} \cong \mathbb{P}^{1}$.

Dimension 5. The story is essentially the same: the cases $(g, n)=(3,0)$ and $(3,1)$ follow from [Fb1, $\mathrm{Fb} 2]$; in other cases we have boundary strata, and classes arising from $\psi_{1}$ on $\overline{\mathcal{M}}_{2, j}(1 \leq j \leq 3)$.

Dimension 6. Here, the first uncertainty arises: one possibility is a codimension 2 class on $\overline{\mathcal{M}}_{3,2} \times \prod \overline{\mathcal{M}}_{0,3}$. This class a priori needn't be tautological on $\overline{\mathcal{M}}_{3,2}$, and $A^{2}\left(\overline{\mathcal{M}}_{3,2}\right)$ has not yet been computed. (There is no technical obstruction to this computation. Presumably $R^{2}\left(\overline{\mathcal{M}}_{3,2}\right)=A^{2}\left(\overline{\mathcal{M}}_{3,2}\right)$.)

One would expect that the relations between these generators of the low dimensional tautological groups will be generated by relations in low genus. For example, they are generated by the cross-ratio relations in dimension 0 and 1 (Section [5.5] and [GrVa3] respectively), and they are likely generated by the cross-ratio relations and Getzler's relation [Ge1] in dimension 2.

5.12. Consequences in low genus. Many interesting consequences of Theorem $\star$ in low genus already follow from Getzler's conjecture and known facts about the moduli space. For example, in genus 1, Corollary 5.1 immediately shows that the tautological groups are generated by boundary strata; but this follows from the classical formula $\psi_{1}=\delta_{0} / 12$. In genus 2, Corollary 5.1 implies that the tautological groups are generated by boundary strata, and the divisor corresponding to $\left.\psi_{1}\right|_{\overline{\mathcal{M}}_{2, n}}$ on the strata corresponding to gluing $\overline{\mathcal{M}}_{2, n}$ to copies of $\overline{\mathcal{M}}_{0, n_{i}}$ and $\overline{\mathcal{M}}_{1, n_{j}}$. This follows from Mumford's formula for $\psi_{1}^{2}$ on $\overline{\mathcal{M}}_{2,1}$ and Getzler's formula for $\psi_{1} \psi_{2}$ on $\overline{\mathcal{M}}_{2,2}$ (equs. (4) and (5) of [Ge2]). 
Conversely, Theorem $\star$ (and even Getzler's conjecture) provides a straightforward proof of both Mumford's and Getzler's formulas. For example, from Theorem $\star$, on $\overline{\mathcal{M}}_{2,1}, \psi_{1}^{2}$ is the sum of boundary strata, and five test families determine the multiplicities with which they occur.

\section{REFERENCES}

[ACo] E. Arbarello and M. Cornalba, The Picard groups of the moduli spaces of curves, Topology 26 (1987), 153-171.

[BFn] K. Behrend and B. Fantechi, The intrinsic normal cone, Invent. Math. 128 (1997), 45-88.

[CH] L. Caporaso and J. Harris, Counting plane curves of any genus, Invent. Math. 131 (1998), 345-392.

[Co] M. Cornalba, On the projectivity of the moduli spaces of curves, J. Reine Angew. Math. 443 (1993), 11-20.

[D] S. Diaz, Complete subvarieties of the moduli space of smooth curves, in Algebraic geometry - Bowdoin 1985 (S. Bloch, ed.), Proc. Sympos. Pure Math. 46, Part 1, Amer. Math. Soc., Providence, RI, 1987, pp. 77-81.

[ELSV1] T. Ekedahl, S. Lando, M. Shapiro, and A. Vainshtein, On Hurwitz numbers and Hodge integrals, C. R. Acad. Sci. Paris Sér. I Math. 328 (1999), 1175-1180.

[ELSV2] T. Ekedahl, S. Lando, M. Shapiro, and A. Vainshtein, Hurwitz numbers and intersections on moduli spaces of curves, Invent. Math. 146 (2001), 297-327.

[Fb1] C. Faber, Chow rings of moduli spaces of curves I: The Chow ring of $\overline{\mathcal{M}}_{3}$, Ann. of Math. (2) 132 (1990), no. 2, 331-419.

[Fb2] C. Faber, Chow rings of moduli spaces of curves II: Some results on the Chow ring of $\overline{\mathcal{M}}_{4}$, Ann. of Math. (2) 132 (1990), no. 3, 421-449.

[Fb3] C. Faber, A conjectural description of the tautological ring of the moduli space of curves, in Moduli of Curves and Abelian Varieties, Aspects Math. E33, Vieweg, Braunschweig, 1999, pp. 109-129.

[FbLo] C. Faber and E. Looijenga, Remarks on moduli of curves, in Moduli of curves and abelian varieties, Aspects Math. E33, Vieweg, Braunschweig, 1999, pp. 23-45.

[FbPa1] C. Faber and R. Pandharipande, Logarithmic series and Hodge integrals in the tautological ring, Michigan Math. J. (Fulton volume) 48 (2000), 215-252.

[FbPa2] C. Faber and R. Pandharipande, Relative maps and tautological classes, J. Eur. Math. Soc. to appear, math.AG/0304485

[FnPa] B. Fantechi and R. Pandharipande, Stable maps and branch divisors, Compositio Math. 130 (2002), no. 3, 345-364.

[FuPa] W. Fulton and R. Pandharipande, Notes on stable maps and quantum cohomology, in Algebraic Geometry - Santa Cruz 1995, Proc. Sympos. Pure Math. 62, Part 2, Amer. Math. Soc., Providence, RI, 1997, pp. 45-96.

[Ga1] A. Gathmann, Absolute and relative Gromov-Witten invariants of very ample hypersurfaces, Duke Math. J. 115 (2002), no. 2, 171-203.

[Ga2] A. Gathmann, manuscript in preparation.

[Ge1] E. Getzler, Intersection theory on $\overline{\mathcal{M}}_{1,4}$ and elliptic Gromov-Witten invariants, J. Amer. Math. Soc. 10 (1997), no. 4, 973-998.

[Ge2] E. Getzler, Topological recursion relations in genus 2, in Integrable systems and algebraic geometry (Kobe/Kyoto, 1997), World Sci. Publishing, River Edge, NJ, 1998, pp. 73-106.

[GrPa1] T. Graber and R. Pandharipande, Localization of virtual classes, Invent. Math. 135, 487-518 (1999).

[GrPa2] T. Graber and R. Pandharipande, Constructions of nontautological classes on moduli spaces of curves, Michigan Math. J. 51 (2003), no. 1, 93-109.

[GrVa1] T. Graber and R. Vakil, Hodge integrals and Hurwitz numbers via virtual localization, Compositio Math. 135 (1) (January 2003), 25-36.

[GrVa2] T. Graber and R. Vakil, On the tautological ring of $\overline{\mathcal{M}}_{g, n}$, in Proceedings of the Seventh Gökova Geometry-Topology Conference 2000, International Press, 2000.

[GrVa3] T. Graber and R. Vakil, work in progress. 
[HaLo] R. Hain and E. Looijenga, Mapping class groups and moduli spaces of curves, in Algebraic Geometry — Santa Cruz 1995, Proc. Sympos. Pure Math. 62, Part 2, Amer. Math. Soc., Providence, RI, 1997, pp. 97-142.

[HMo] J. Harris and I. Morrison, Moduli of Curves, GTM 187, Springer-Verlag, New York, 1998.

[I] E.-N. Ionel, Topological recursive relations in $H^{2 g}\left(M_{g, n}\right)$, Invent. Math. 148 (2002), no. 3, 627-658.

[IP1] E.-N. Ionel and T. Parker, Relative Gromov-Witten invariants, Ann. of Math. (2) 157 (2003), 45-96.

[IP2] E.-N. Ionel and T. Parker, The symplectic sum formula for Gromov-Witten invariants, Ann. of Math. (2), to appear.

[Ka] E. Katz, Ph.D. thesis, Stanford University, 2004.

[Ke] S. Keel, Intersection theory of moduli space of stable n-pointed curves of genus 0, Trans. Amer. Math. Soc. 330 (1992), 545-574.

[Li1] J. Li, Stable morphisms to singular schemes and relative stable morphisms, J. Diff. Geom. 57 (2001), no. 3, 509-578.

[Li2] J. Li, A degeneration formula of GW-invariants, J. Diff. Geom. 60 (2002), no. 2, 199-293.

[LR] A.-M. Li and Y. Ruan, Symplectic surgery and Gromov-Witten invariants of Calabi 3-folds, Invent. Math. 145 (2001), no. 1, 151-218.

[LLZ1] C.-C. M. Liu, K. Liu, and J. Zhou, On a proof of a conjecture of Marino-Vafa on Hodge integrals, Math. Res. Lett. 11 (2004), no. 2, 259-279, math.AG/0306434v1.

[LLZ2] C.-C. M. Liu, K. Liu, and J. Zhou, A proof of a conjecture of Marino-Vafa on Hodge integrals, J. Diff. Geom. 65 (2004), 289-340, math.AG/0306257v1.

[Lo] E. Looijenga, On the tautological ring of $\mathcal{M}_{g}$, Invent. Math. 121 (1995), no. 2, 411-419.

[M] D. Mumford, Toward an enumerative geometry of the moduli space of curves, in Arithmetic and Geometry, Vol. II, M. Artin and J. Tate ed., Prog. Math. 36, Birk. Boston, Boston, MA, 1983, pp. 271-328.

[MFK] D. Mumford, J. Fogarty, and F. Kirwan, Geometric Invariant Theory, Springer-Verlag, Berlin, 1994.

[OPa] A. Okounkov and R. Pandharipande, Virasoro constraints for target curves, preprint 2003, math.AG/0308097

[Pa] R. Pandharipande, Three questions in Gromov-Witten theory, in Proceedings of the ICM, Vol. II, 2002, T. Li et al eds., pp. 503-512.

[Po] M. Polito, The fourth tautological group of $\bar{M}_{g, n}$ and relations with the cohomology, preprint, 2000.

[Va1] R. Vakil, The enumerative geometry of rational and elliptic curves in projective space, J. Reine Angew. Math. 529 (2000), 101-153.

[Va2] R. Vakil, Genus 0 and 1 Hurwitz numbers: Recursions, formulas, and graph-theoretic interpretations, Trans. Amer. Math. Soc. 353 (2001), no. 10, 4025-4038.

[Va3] R. Vakil, The moduli space of curves and its tautological ring, Notices Amer. Math. Soc. 50 (June/July 2003), no. 6, 647-658.

\section{Dept. of Mathematics, University of CALifornia, Berkeley CA}

E-mail address: graber@math . berkeley.edu

DePt. OF MATHEMATICS, STANFORD UniVERSity, StANFORD CA

E-mail address: vakil@math.stanford.edu 